\title{
Supporting Information for Computational Calculation of Dissolved Organic Matter Absorption Spectra
}

\author{
Frank Leresche ${ }^{a, b, \#}$, Elena A. Vialykh ${ }^{\text {a,b.\#, and Fernando L. Rosario-Ortiz }}{ }^{\text {a,b* }}$ \\ a Department of Civil, Environmental, and Architectural Engineering \\ University of Colorado Boulder, CO 80309 \\ ${ }^{\mathrm{b}}$ Environmental Engineering Program \\ University of Colorado Boulder, CO 80309 \\ \# Authors contributed equally to the work \\ ${ }^{*}$ Corresponding author: Frank.Leresche@ colorado.edu
}




\section{Table of Contents}

S3 Table S1. Structures presents in the models before and after sodium borohydride reduction.

S19 Table S2. Characteristics of the SRFA models.

S20 Figure S1. Comparison between the calculated and measured UV-Vis spectra of flavone and coumarin.

S21 Table S3. Structures that were used to investigate intra-molecular charge transfer interactions.

S24 Table S4. Molecules absorption by region

S28 Figure S2. Some of the structures and their corresponding calculated UV-Vis absorbance spectra

S29 Table S5 Absorption maximum ( $\left.\lambda_{\mathrm{a}, \mathrm{max}}\right)$ of calculated spectra for a series of flavones substituted molecules.

S30 Text S1. Derivation of the extent of reaction formula for the estimation of inter molecular charge transfer interactions.

S30 Table S6. Extent of reaction table.

S30 Table S7. Evaluation of inter-molecular charge transfer interactions.

S32 Figure S3. Additional compounds investigated for unconjugated intra-molecular charge transfer interactions.

S33 Figure S4. Additional flavone and coumarin backboned substituted molecules investigated for intra-molecular charge transfer interactions.

S34 Figure S5. Additional molecules investigated for conjugated intra molecular charge-transfer interactions.

S35 Table S8. Calculated charge-transfer (CT) contribution to the lowest singlet excited state.

S36 Table S9. Effects of sodium borohydride reduction on the absorption maximum $\left(\lambda_{\mathrm{a}, \max }\right)$.

S37 Figure S6. Example of calculation of the chromophoric part of a structure.

S37 Table S10. Evaluation of DOM chromophoric carbon fraction. 
Table S1. Structures presents in the models before and after sodium borohydride reduction.

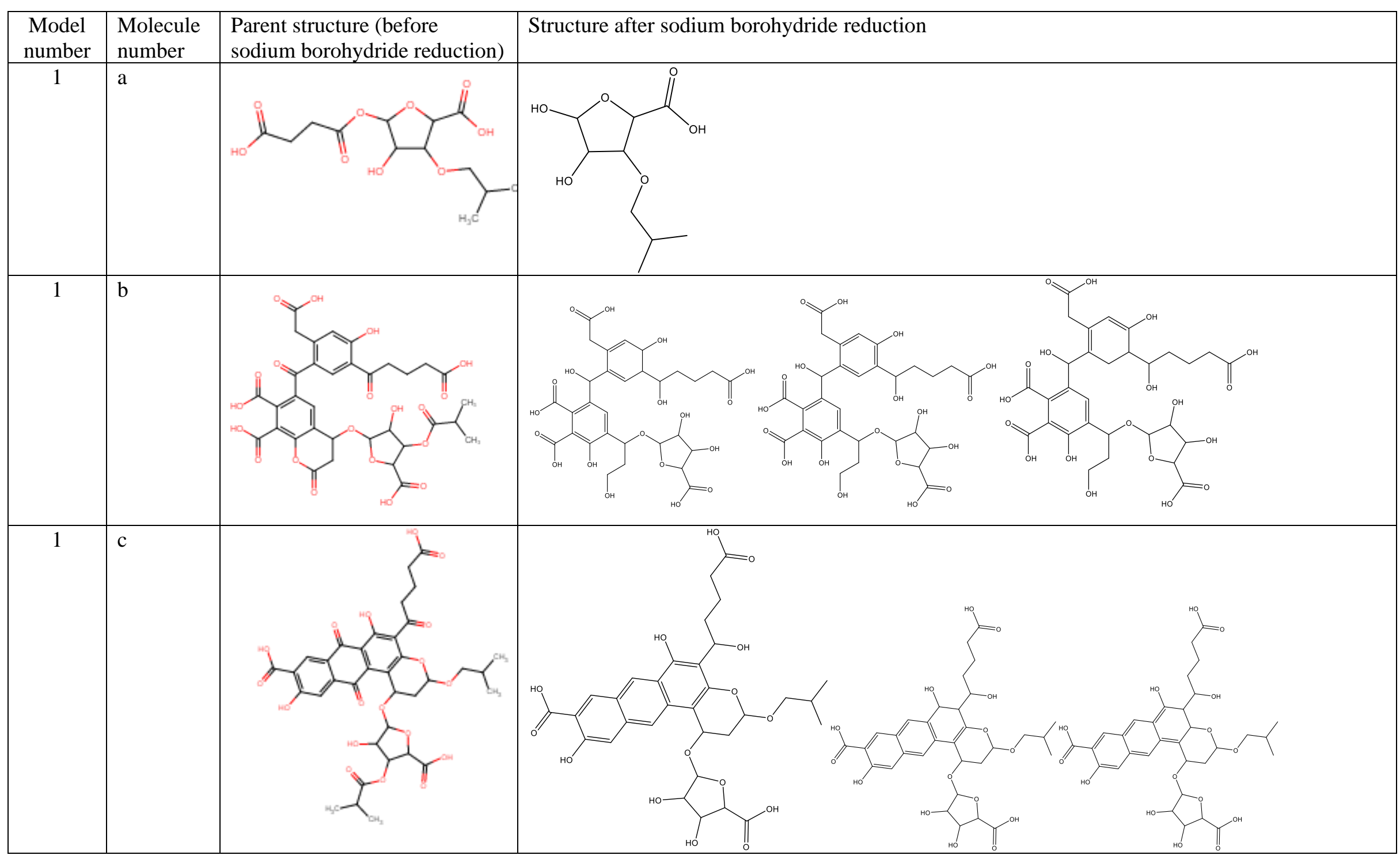




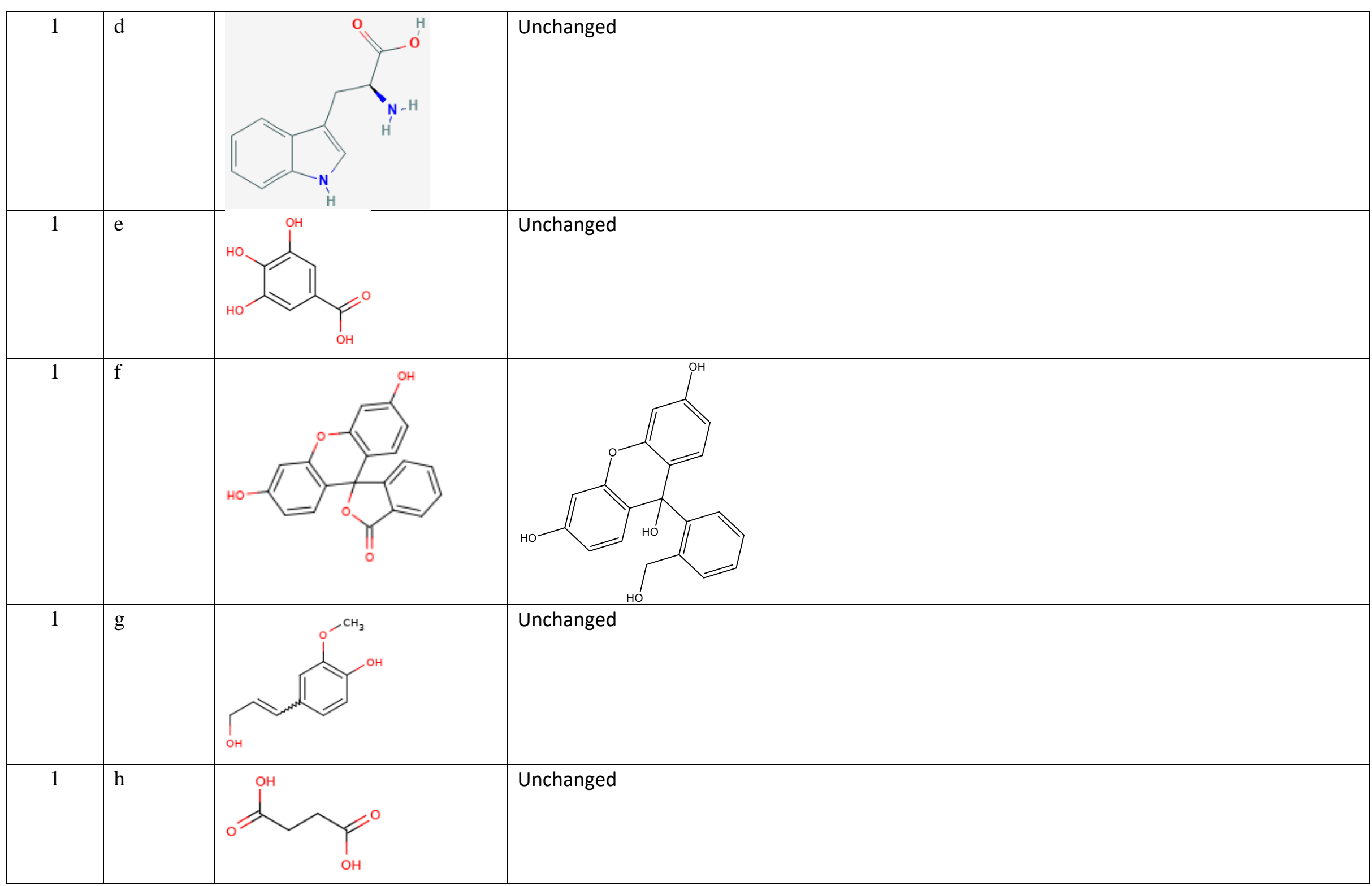




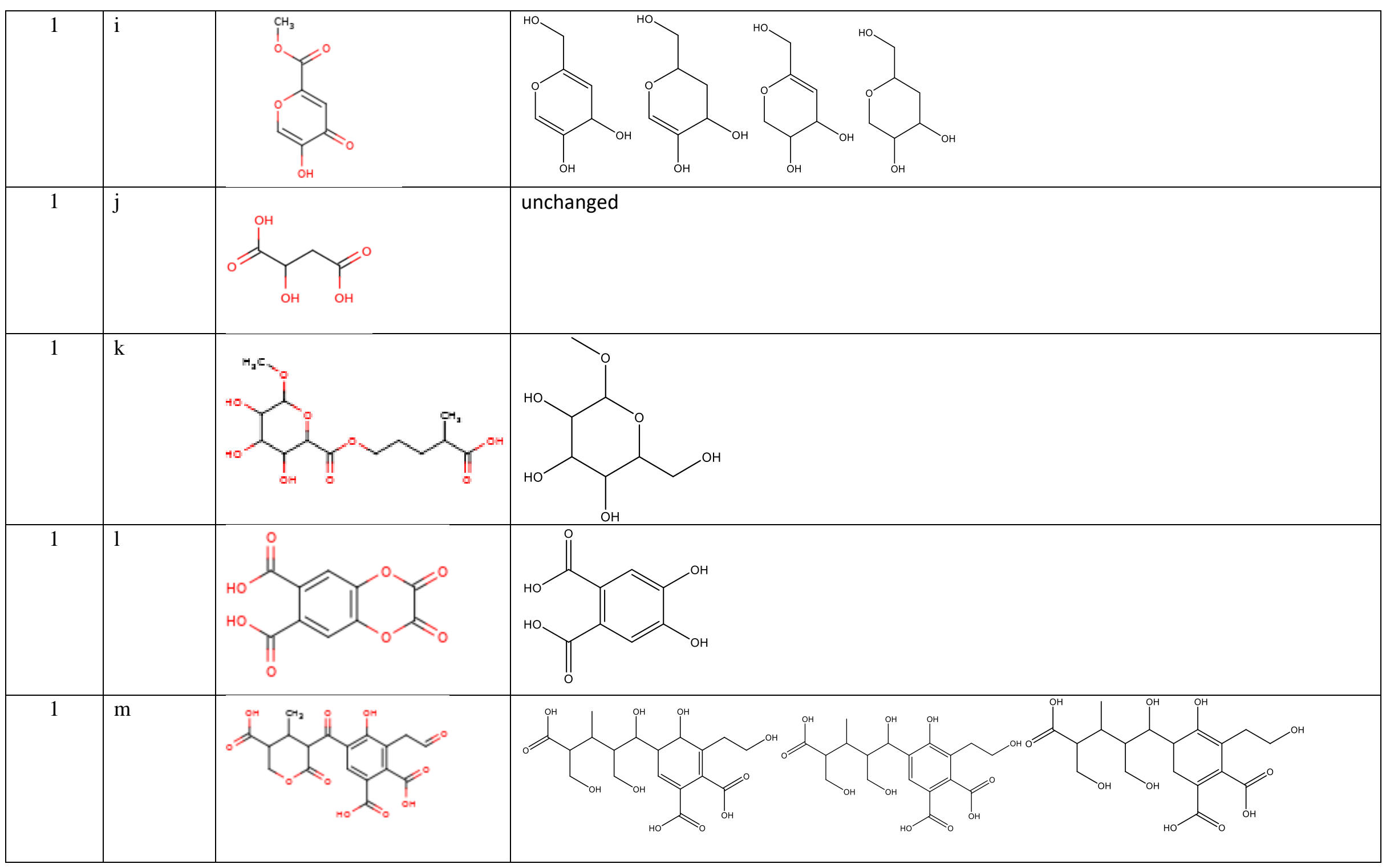




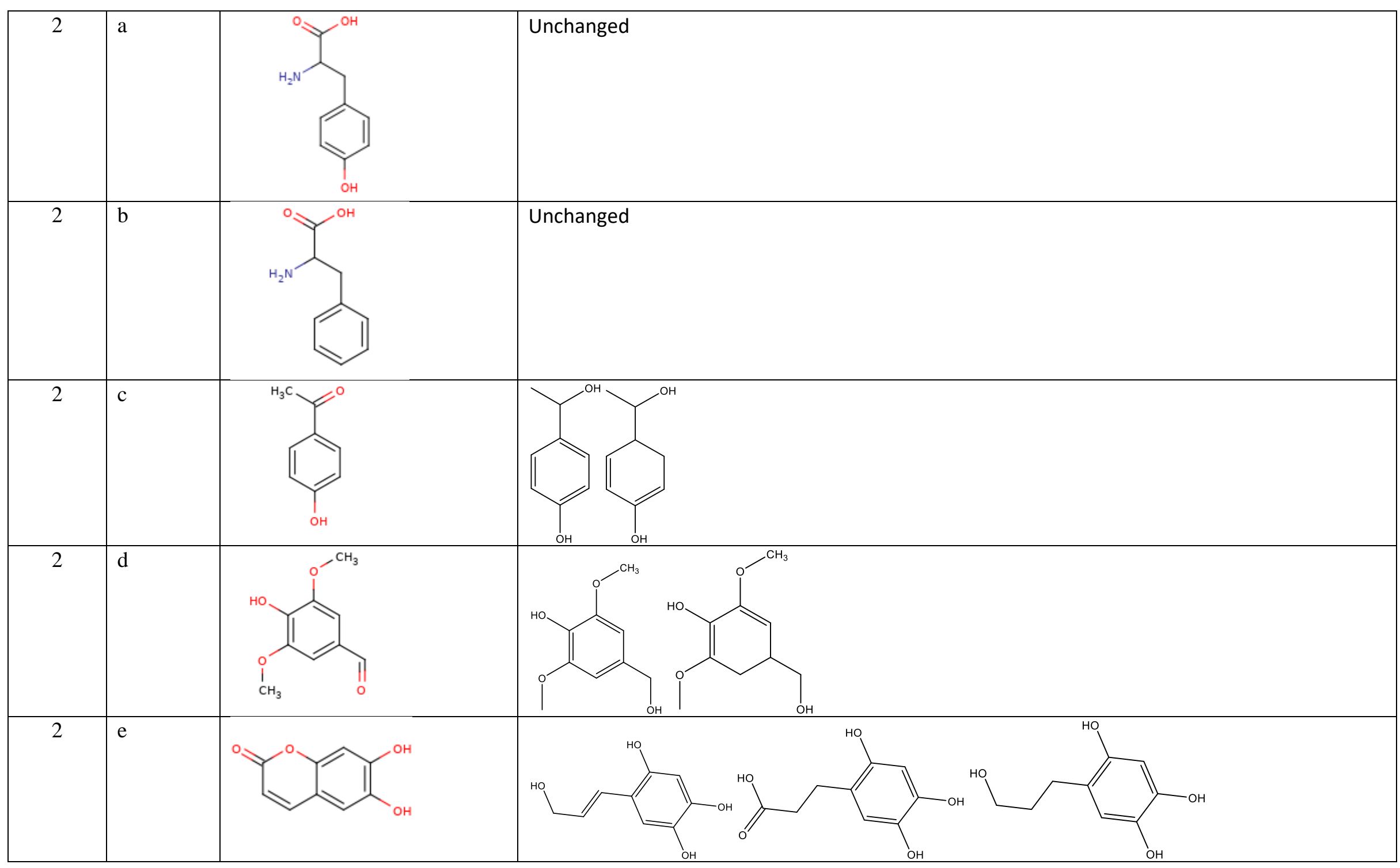




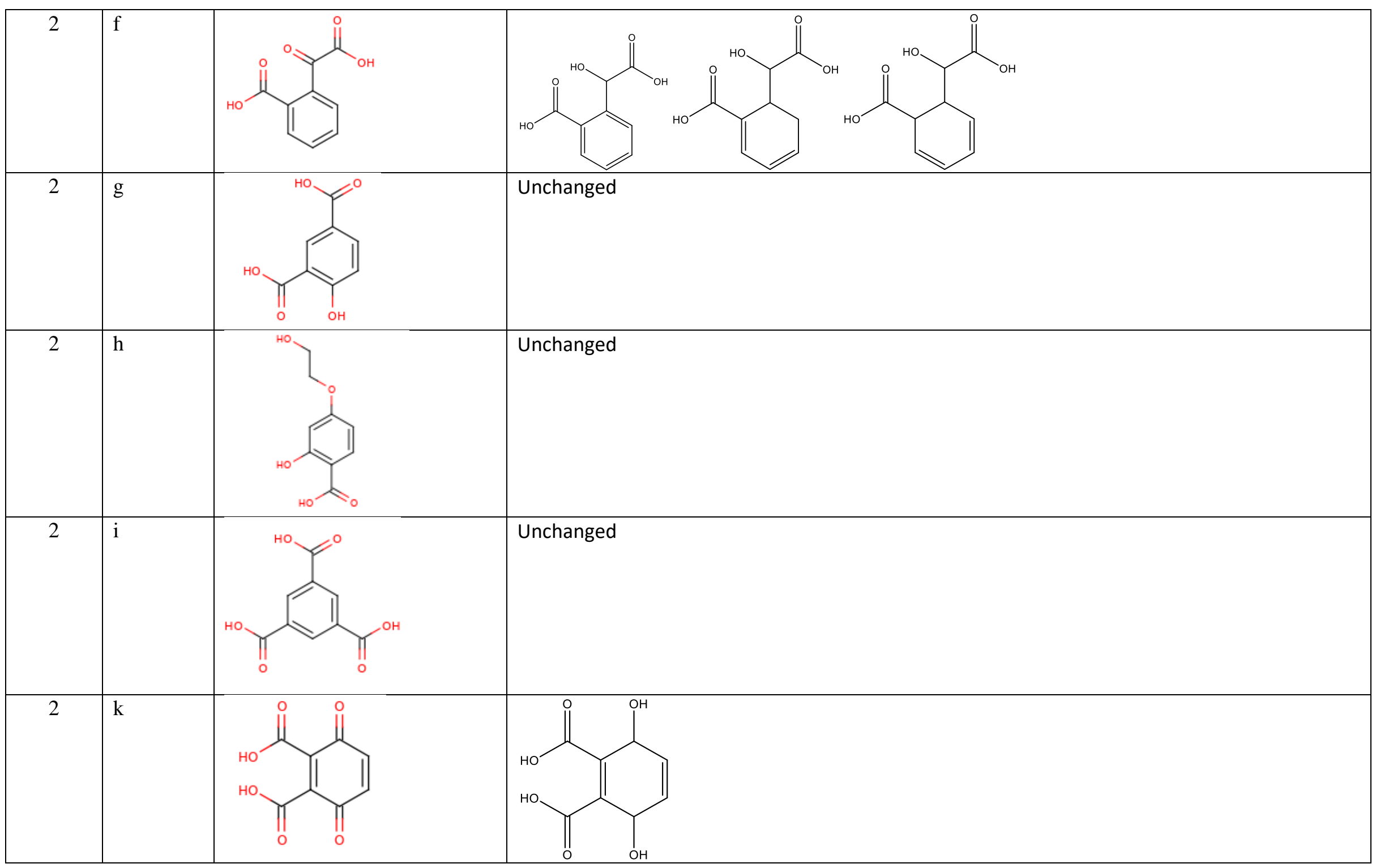




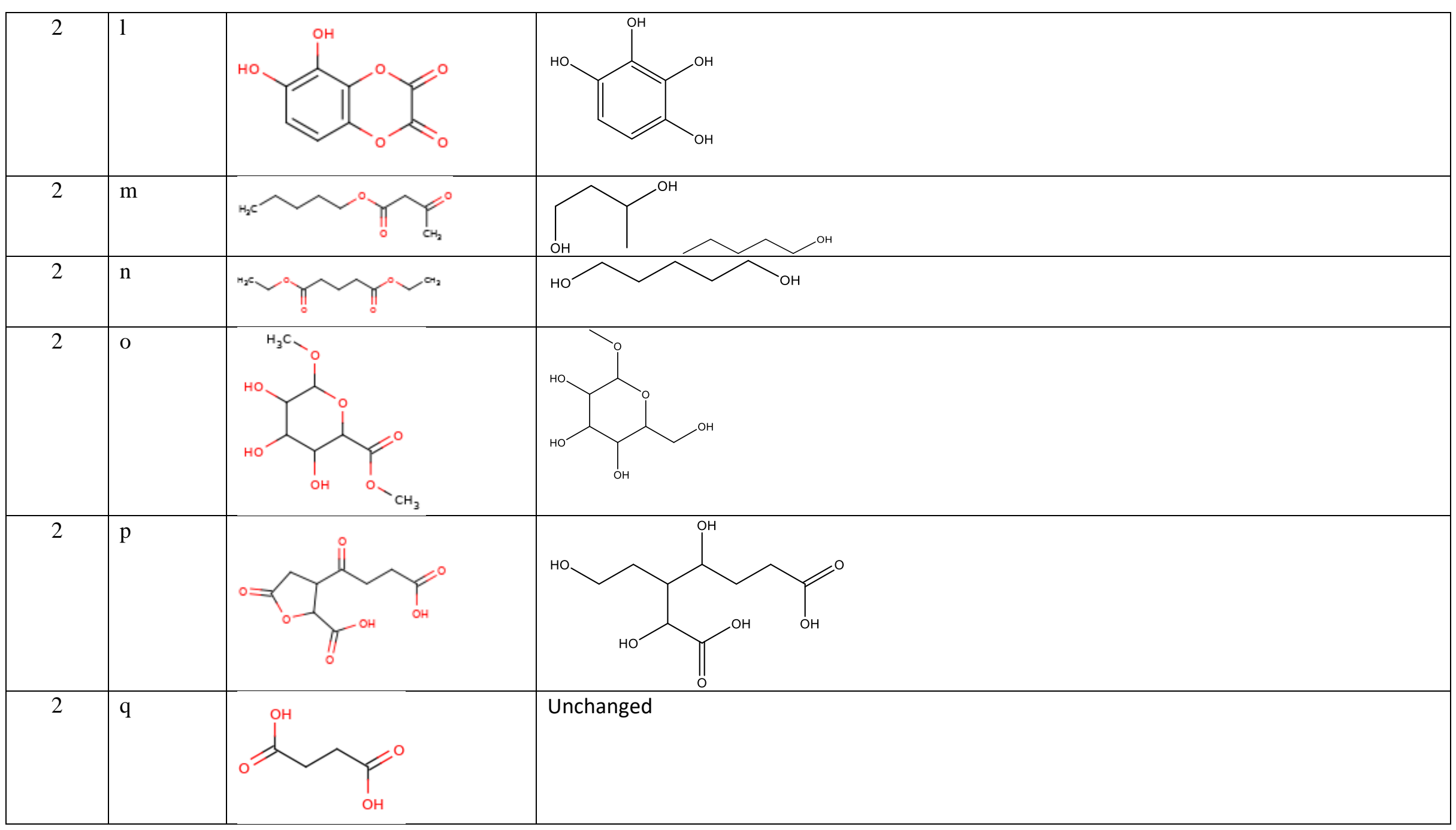




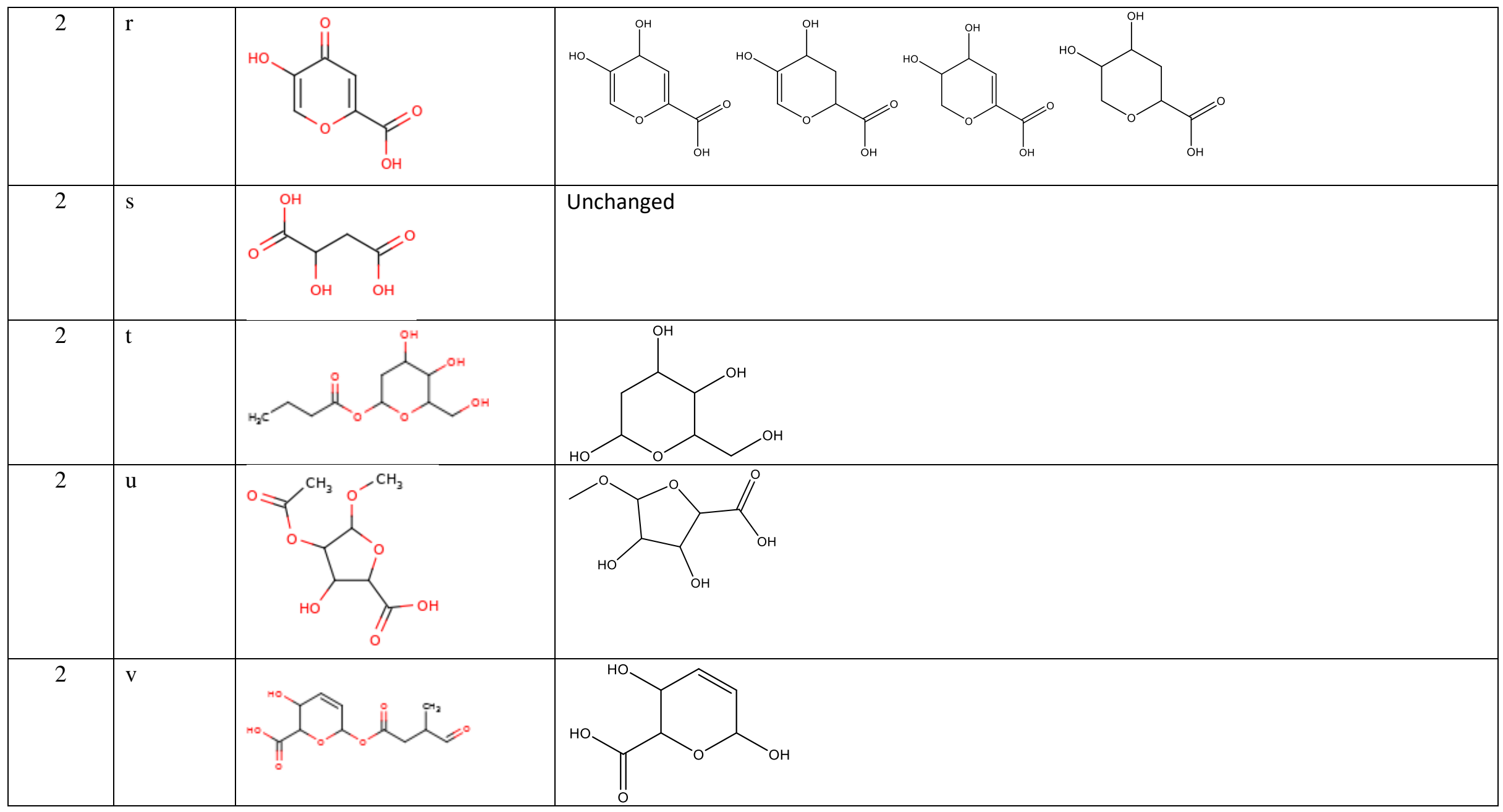

S9 


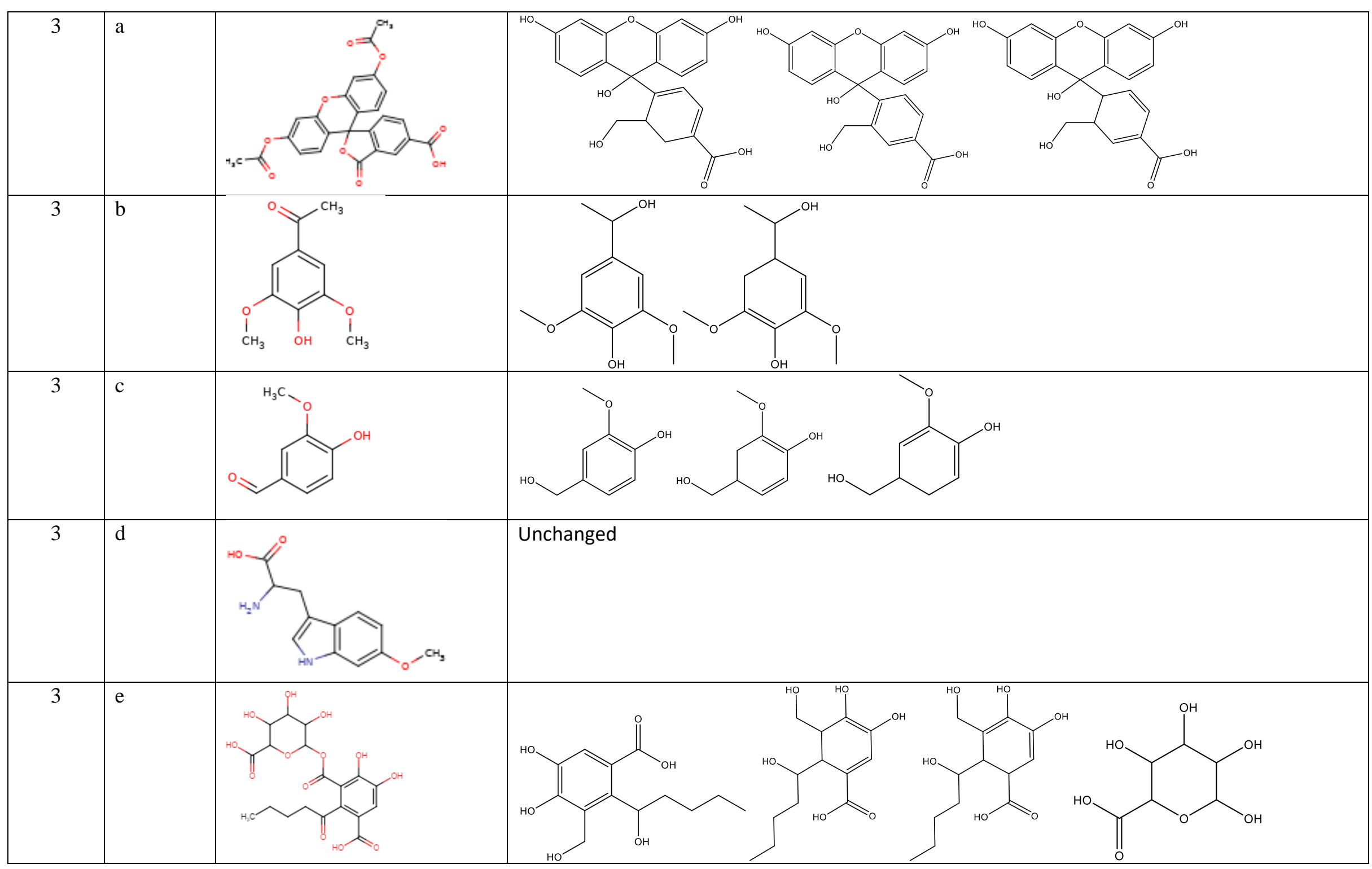


<smiles>CCCCCCCC(=O)C(OC(O)C(O)C(O)C(O)C(=O)O)c1cc(O)cc(C(=O)O)c1C(=O)O</smiles>

S11 


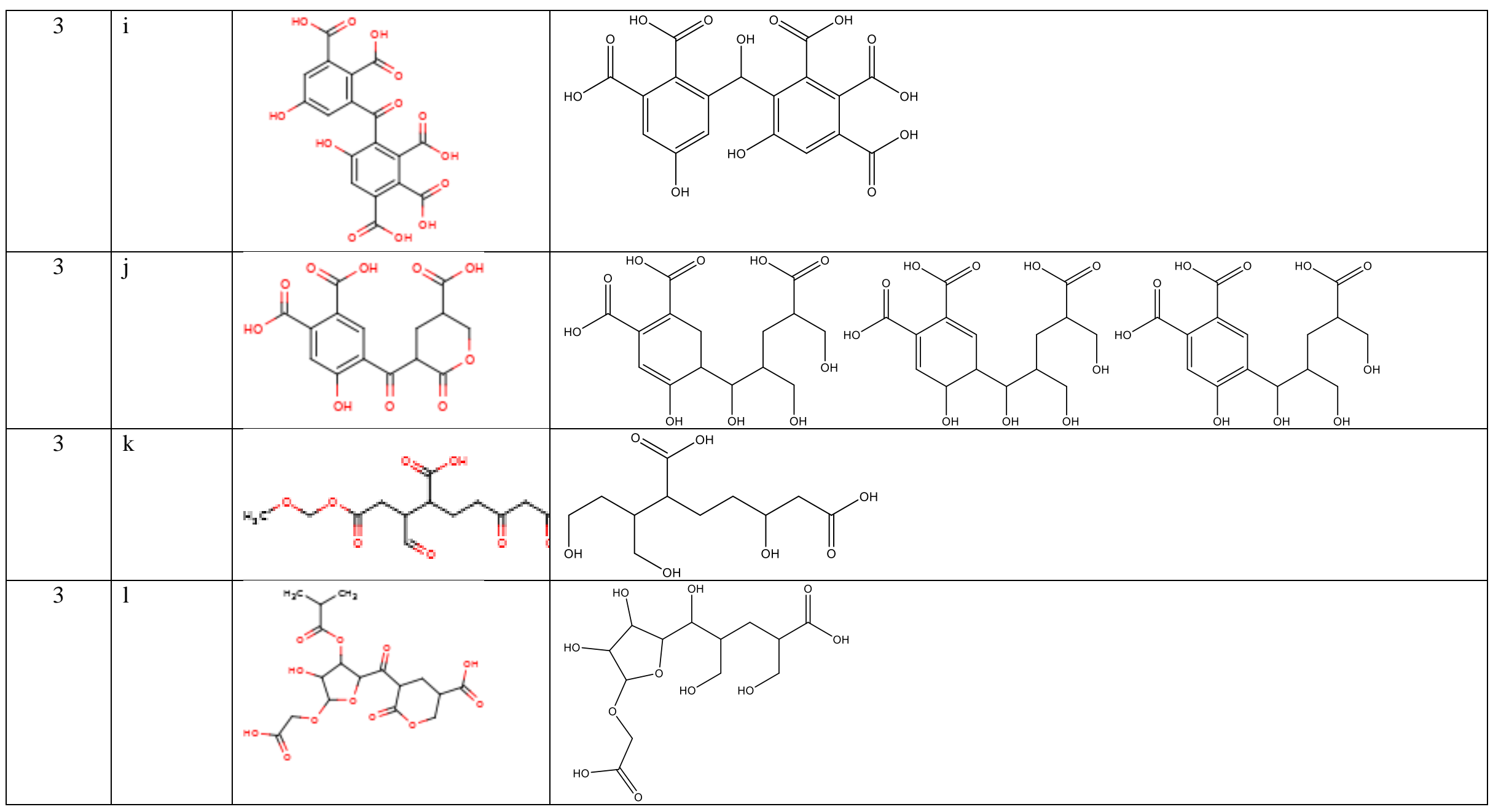




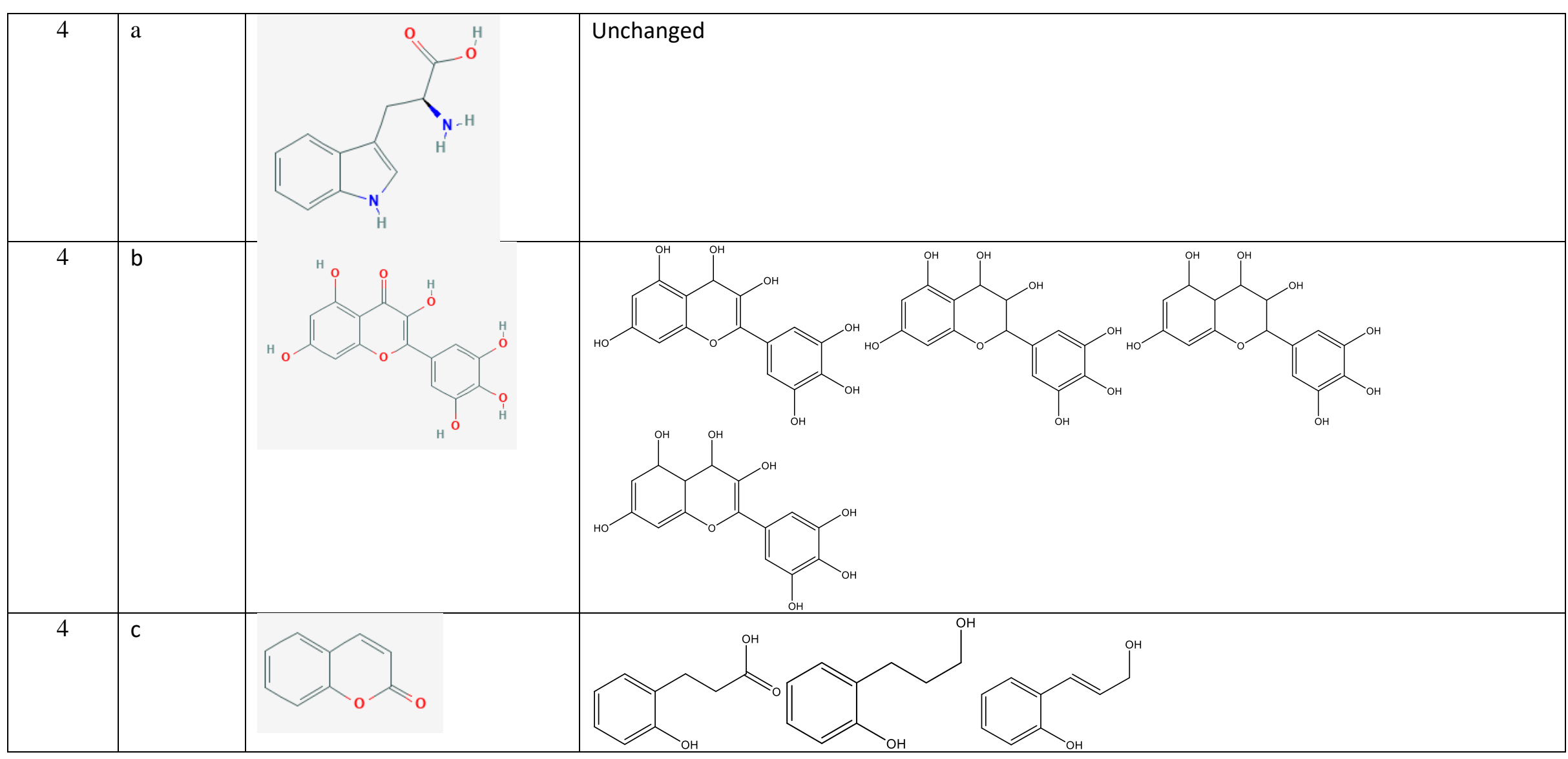




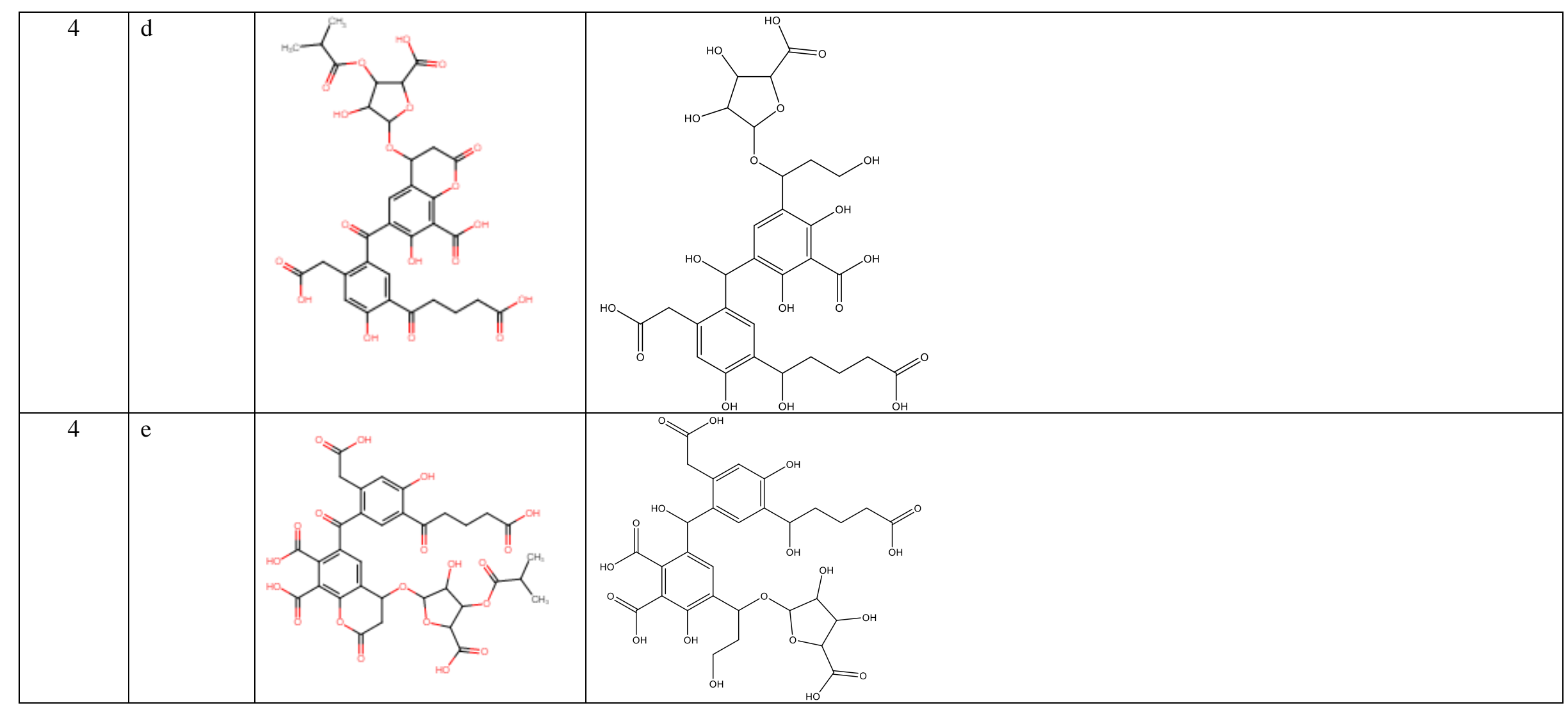




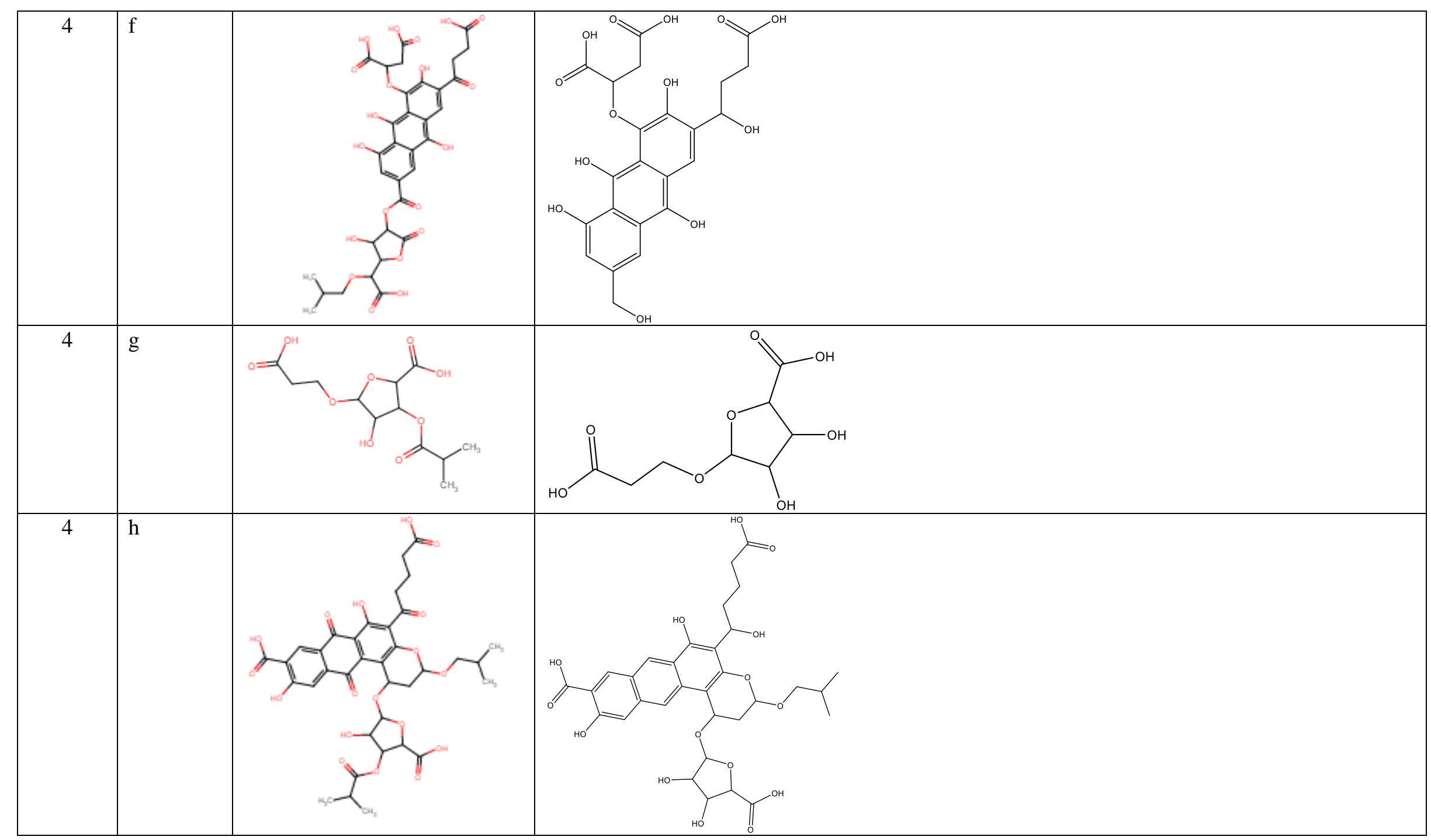




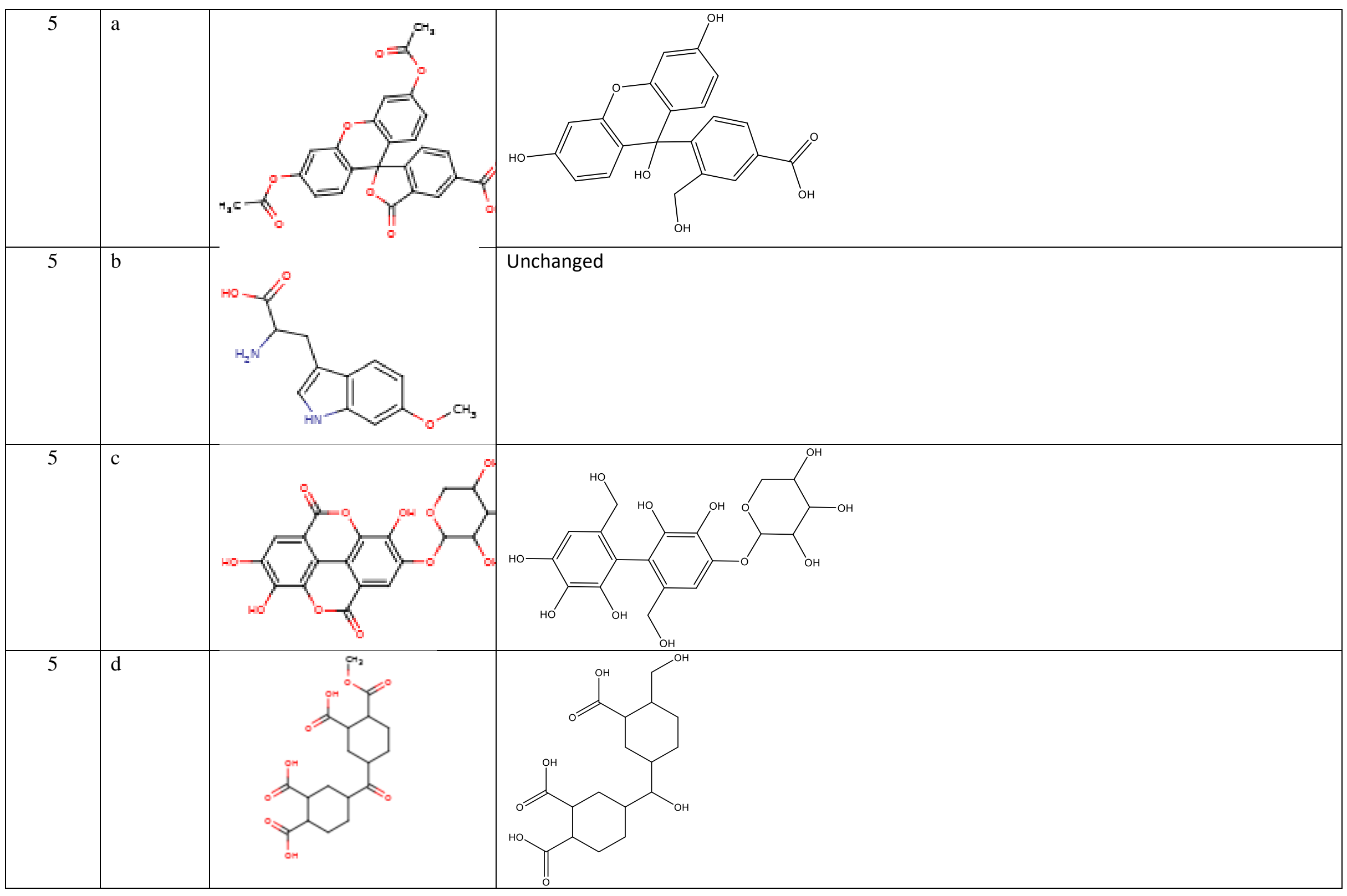




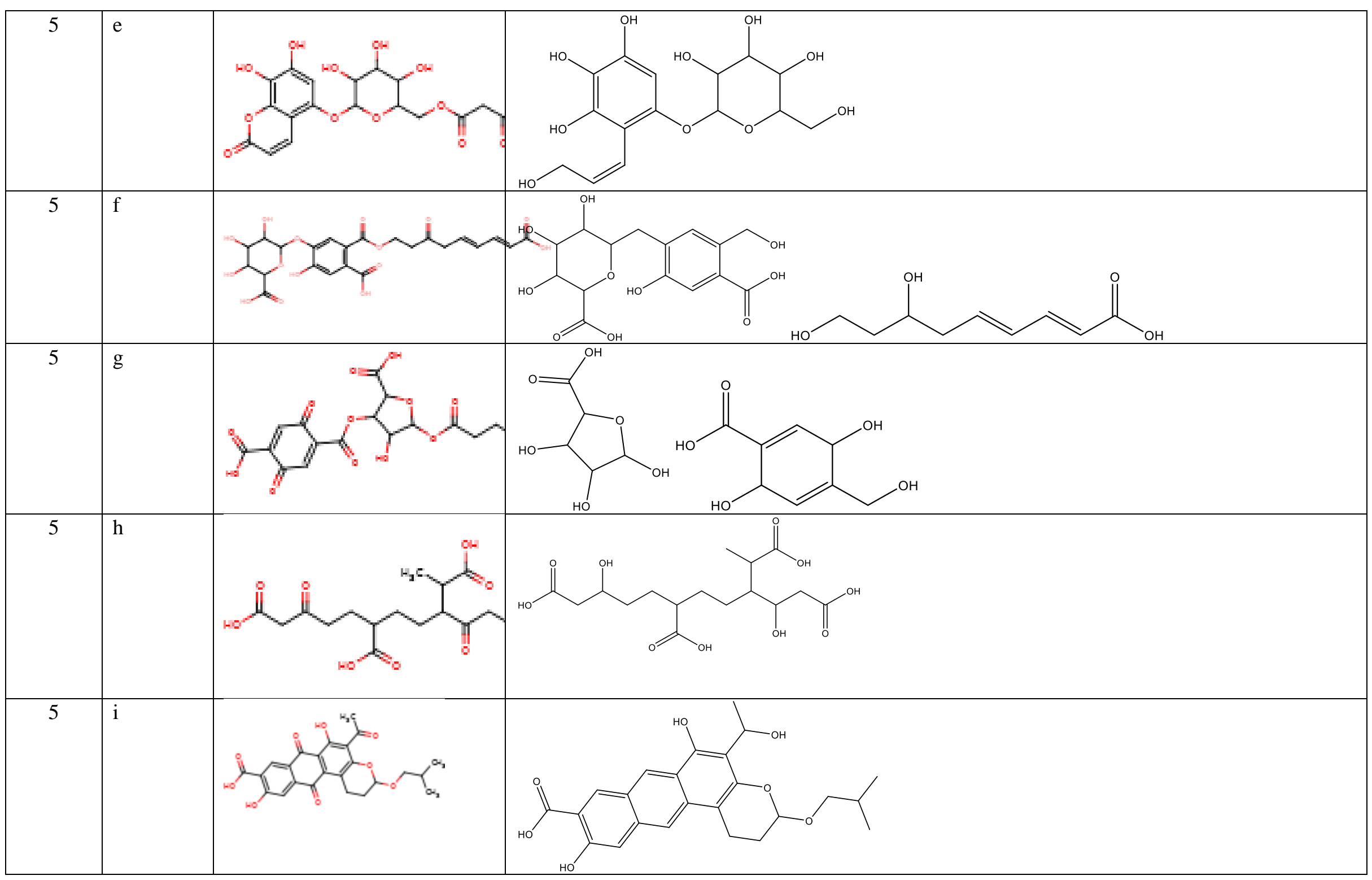




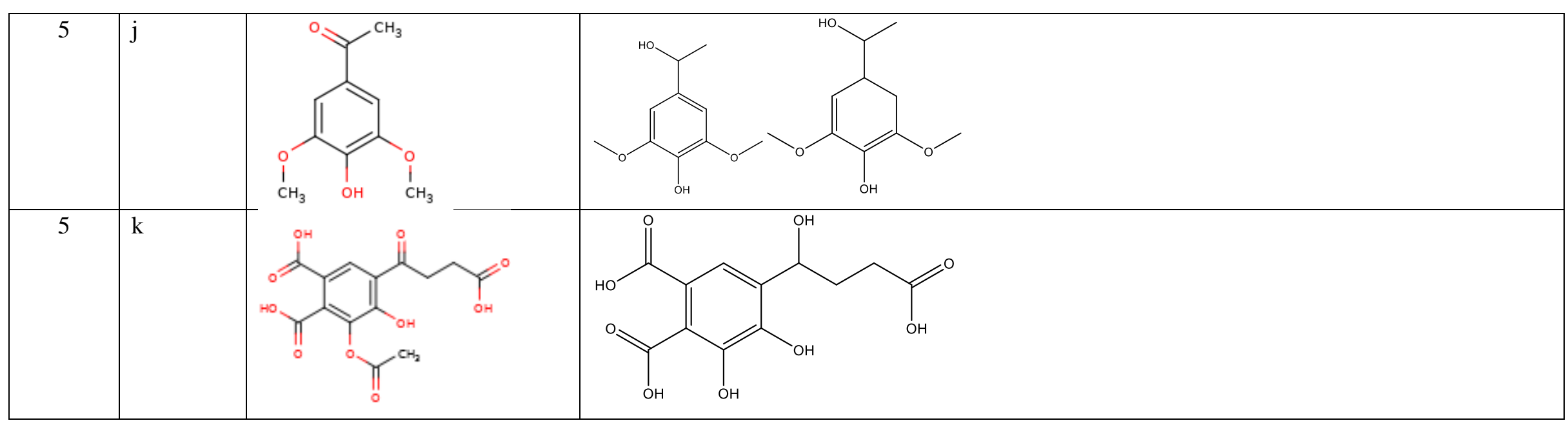


Table S2. Characteristics of the generated models representing SRFA. A total of five models were created for this study, using the methods detailed elsewhere. ${ }^{1}$

\begin{tabular}{|l|c|c|c|c|c|c|c|}
\hline & $\mathbf{C}$ & $\mathbf{H}$ & $\mathbf{O}$ & $\mathbf{N}$ & $\begin{array}{c}\text { Min. size of } \\
\text { molecule, Da }\end{array}$ & $\begin{array}{c}\text { Max. size of } \\
\text { molecule, Da }\end{array}$ & $\begin{array}{c}\text { Mean molecular } \\
\text { weight of model, } \\
\text { Da }\end{array}$ \\
\hline Model 1 & 38.6 & 39.6 & 21.4 & 0.41 & 118 & 760 & 318 \\
\hline Model 2 & 36.3 & 41.6 & 21.6 & 0.42 & 118 & 258 & 188 \\
\hline Model 3 & 38.0 & 39.6 & 22.0 & 0.40 & 152 & 486 & 356 \\
\hline Model 4 & 39.5 & 38.9 & 21.2 & 0.43 & 146 & 760 & 497 \\
\hline Model 5 & 39.4 & 38.6 & 21.7 & 0.40 & 196 & 540 & 391 \\
\hline
\end{tabular}




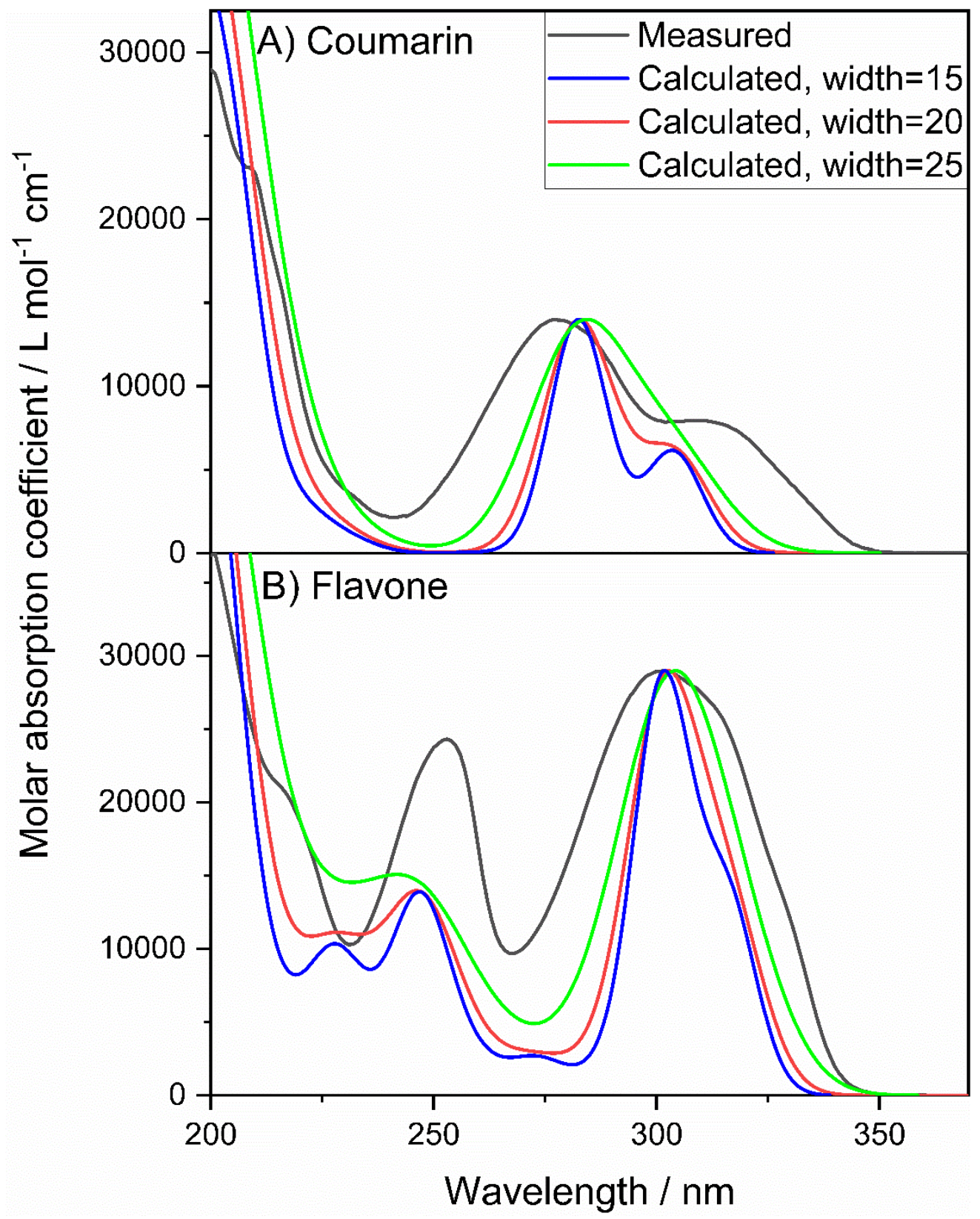

Figure S1. Comparison between the calculated and measured UV-Vis spectra of a) coumarin and b) flavone. The measurements were done in 75/25 Water / Methanol mixture. The calculated spectra absorbance intensity was normalized to the one of the measured spectra using the measured absorption coefficient at $\lambda=277 \mathrm{~nm}$ for coumarin and $\lambda=302 \mathrm{~nm}$ for flavone. For the calculated spectra, the peak width of 15,20 and 25 are presented. This peak width is a parameter that is used to simulate the vibrational transitions. 
Table S3. Structures that were used to investigate non-conjugated intra-molecular charge transfer interactions.

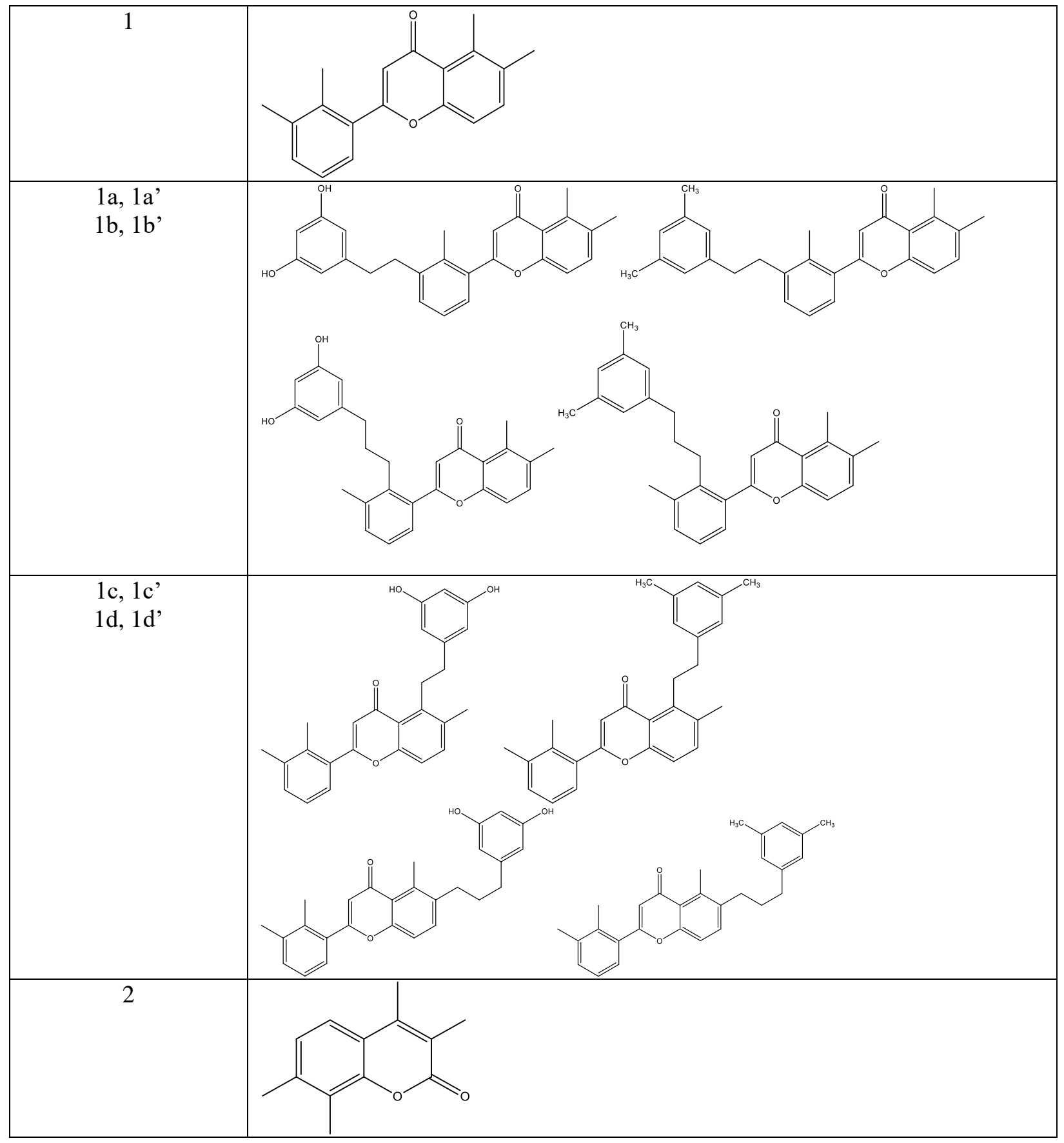




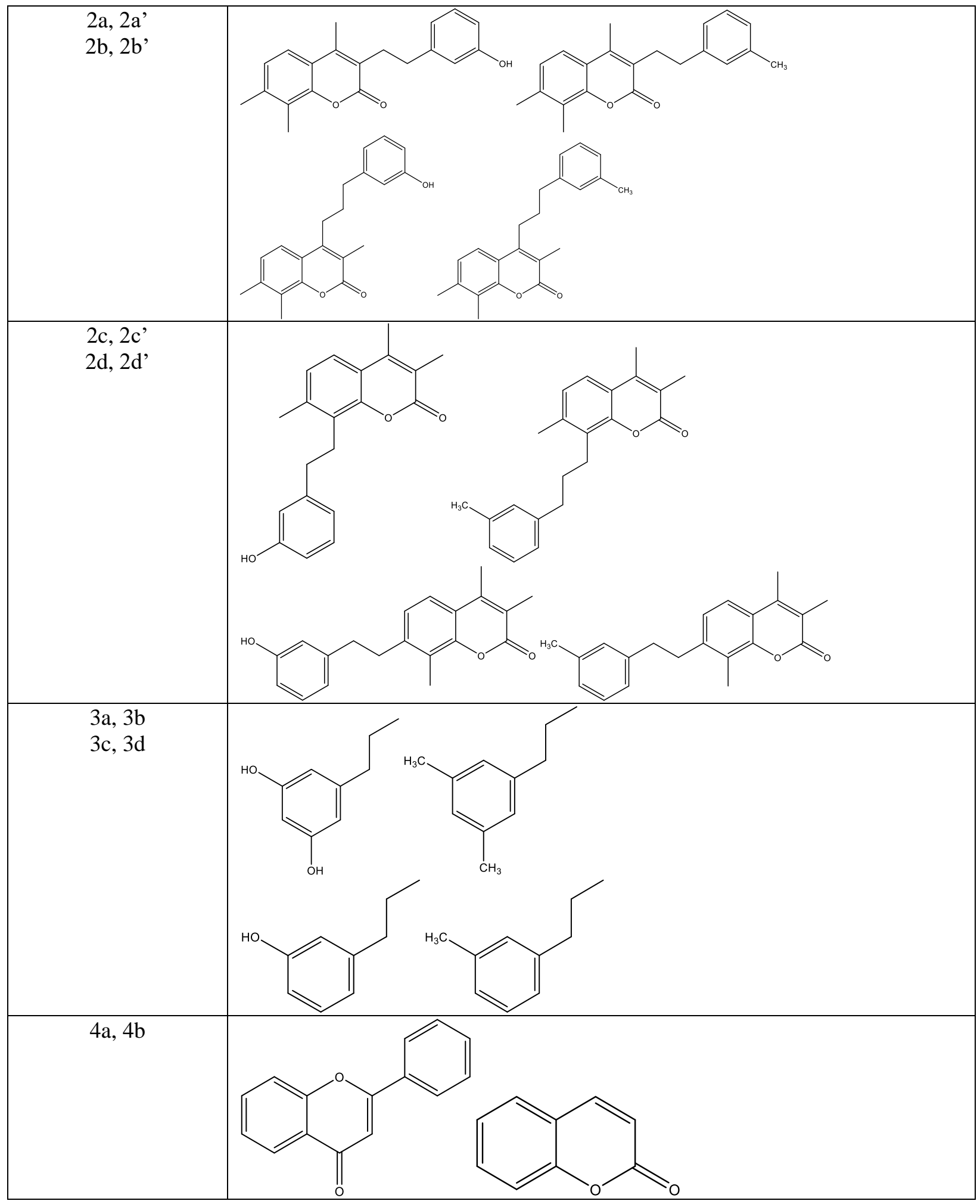




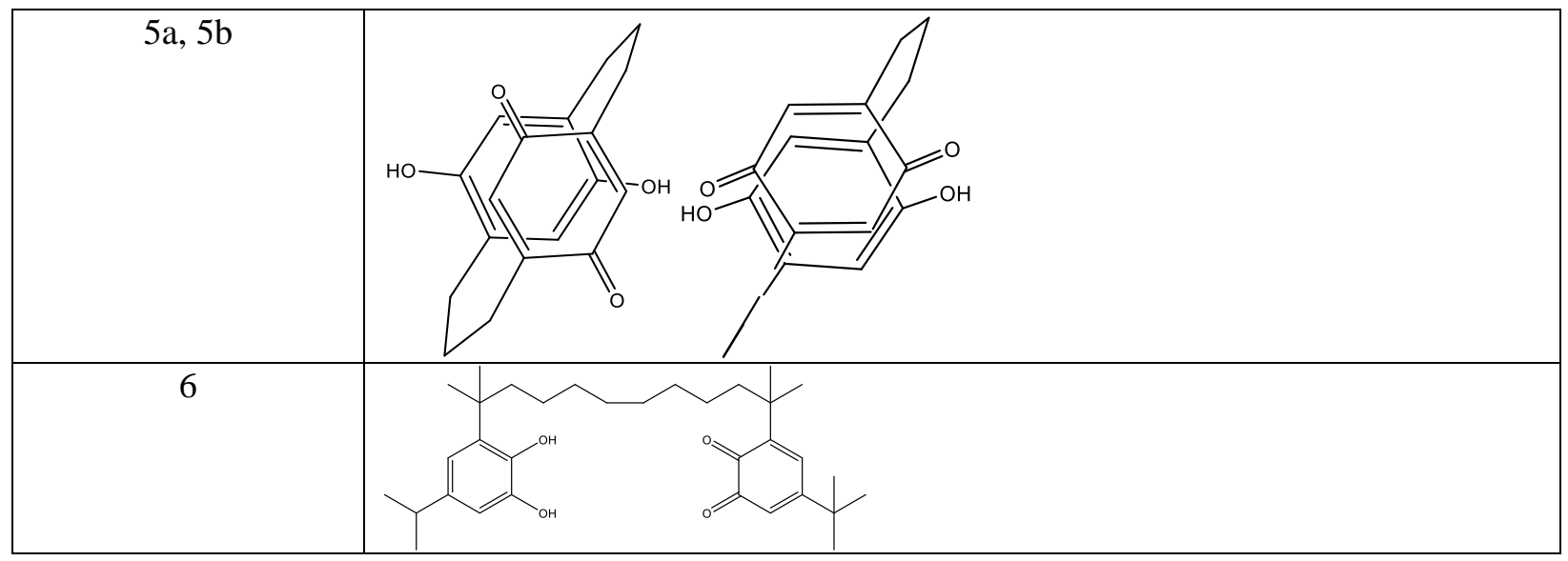


Table S4. Molecules absorption by region

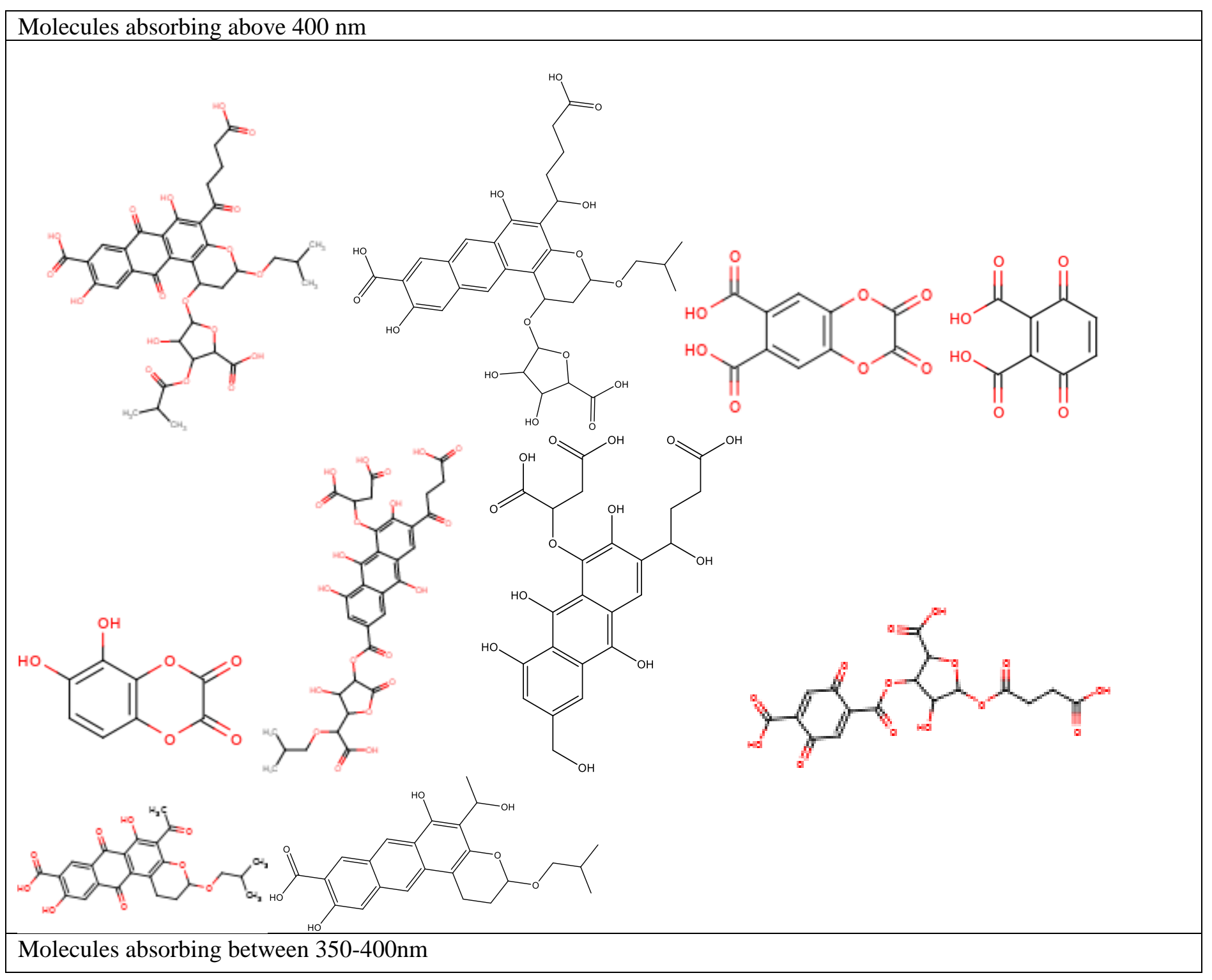




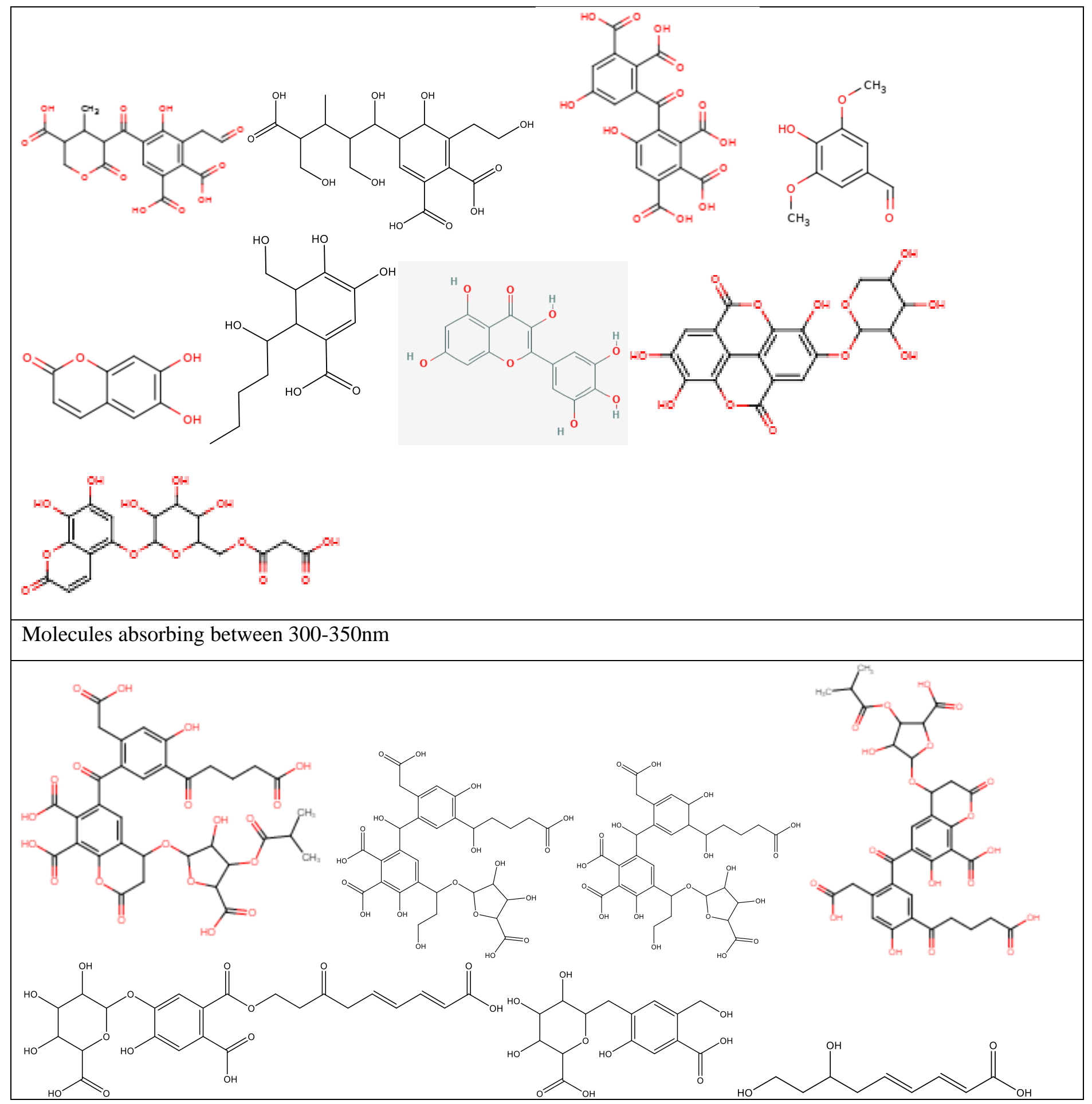




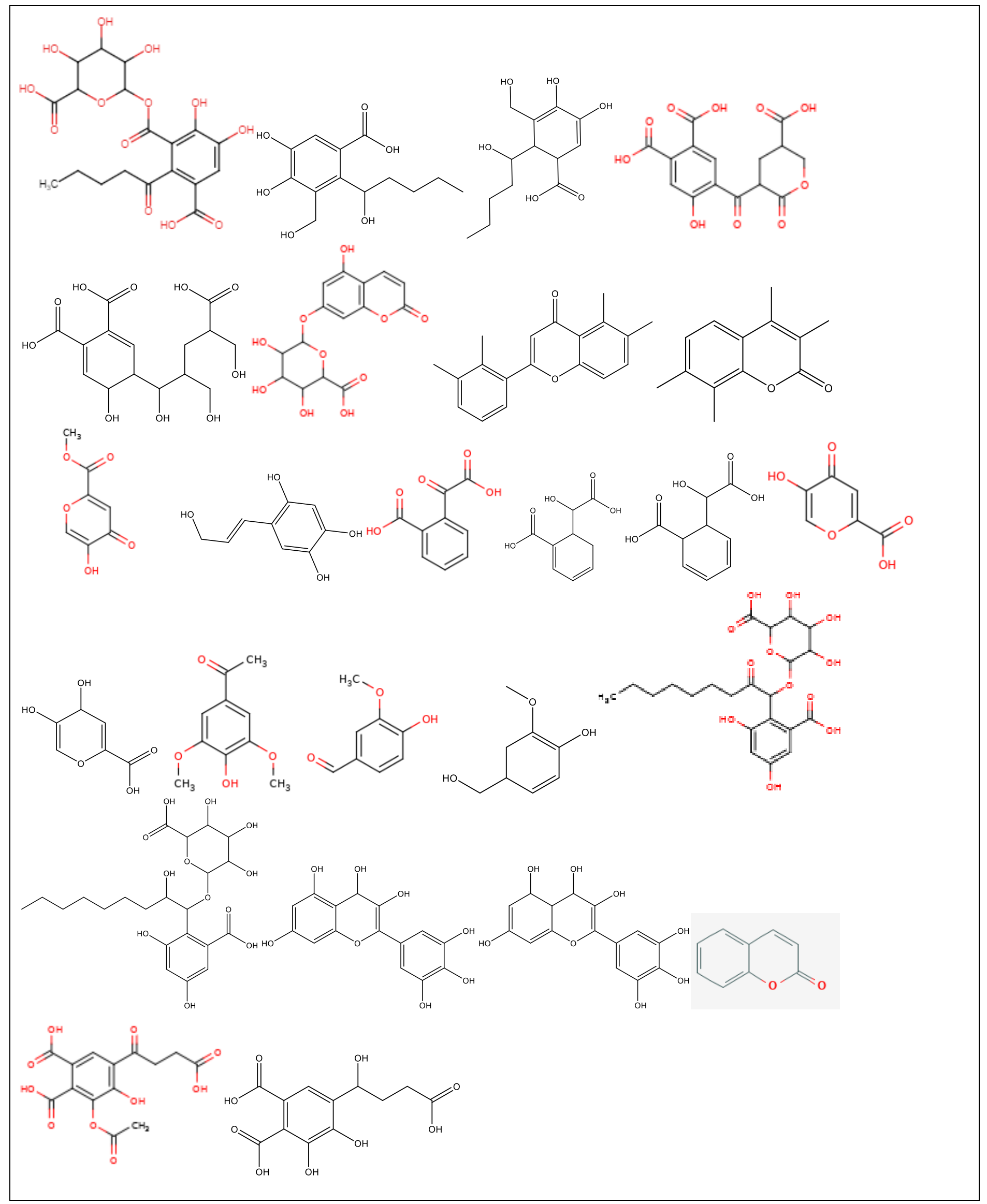




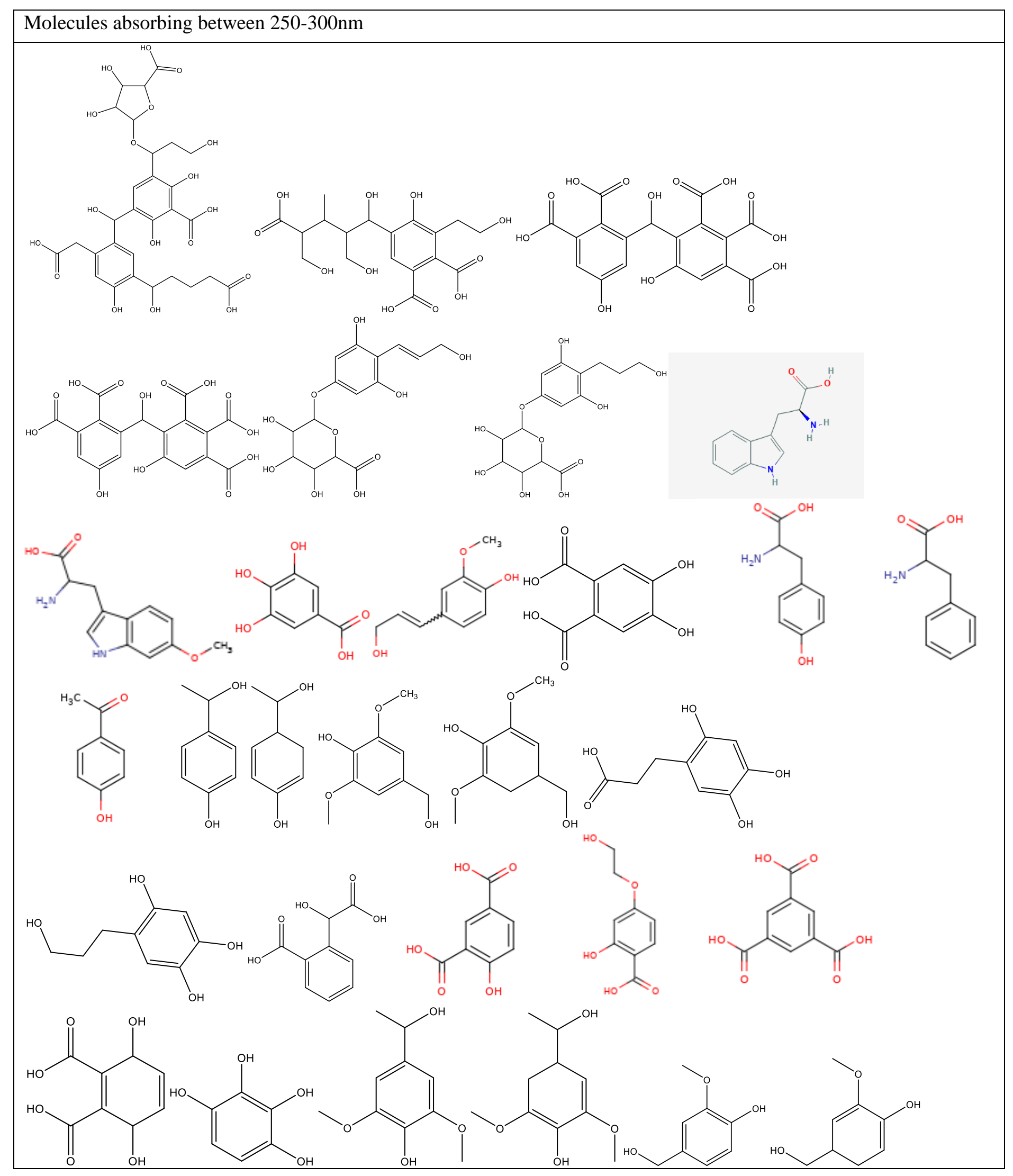



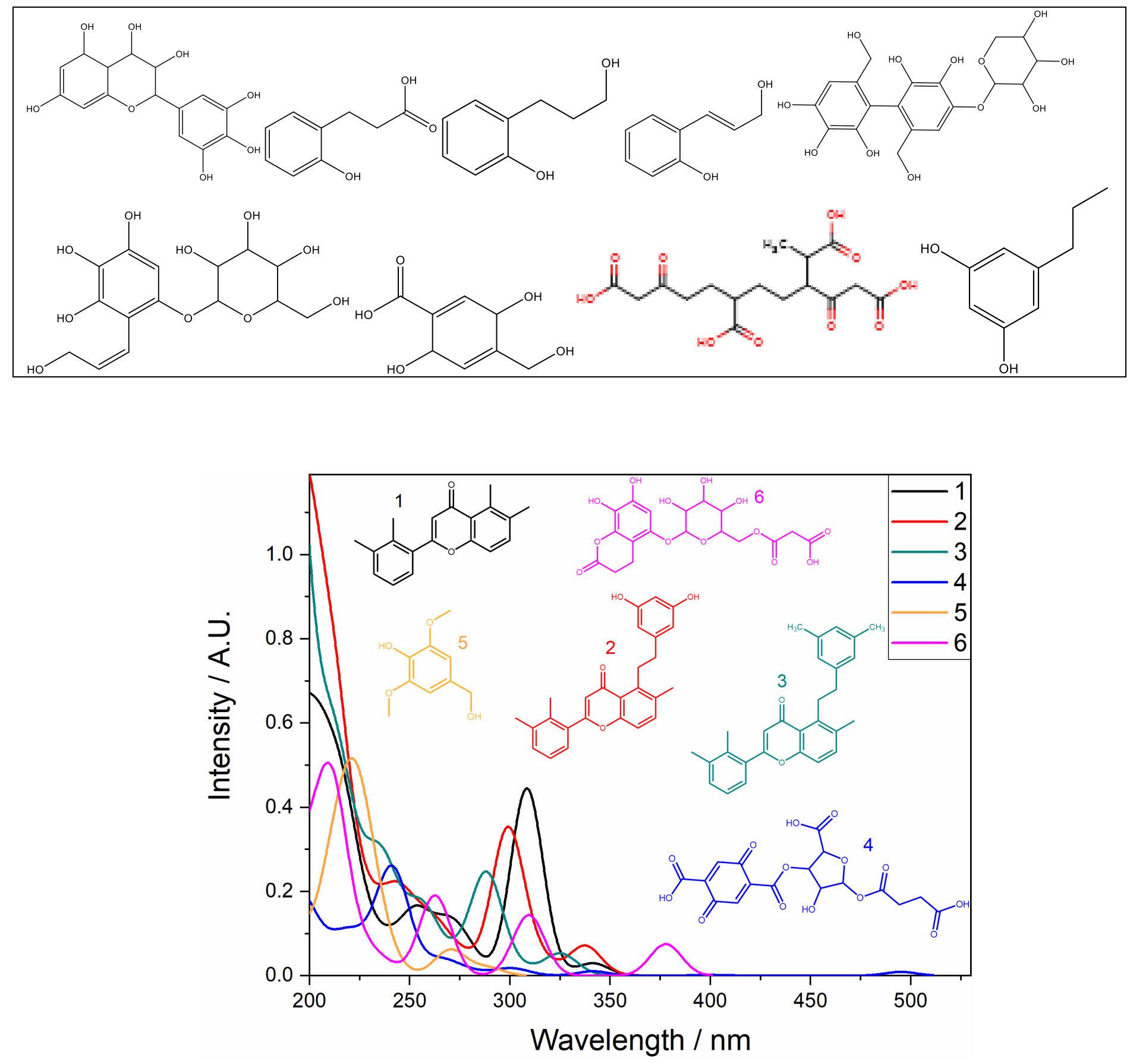

Figure S2. Some of the structures and their corresponding calculated UV-Vis absorbance spectra. The backbone of structure 1, 2 and 3 are based on flavone, structure 4 on quinone, structure 5 on phenol and structure 6 on coumarin. For molecules 4 and 6, the deprotonated form (carboxylic acid) of the molecule's absorbance spectra is shown. 
Table S5. Absorption maximum wavelength $\left(\lambda_{\mathrm{a}, \max }\right)$ and absorption maximum wavelength for the peaks centered around 300nm $\left(\lambda_{\mathrm{M}}\right)$ of calculated spectra for a series of flavones backbone substituted molecules (see Figure S2).

\begin{tabular}{|c|c|c|c|c|c|c|}
\hline $\mathrm{R}=$ & Hammett $\sigma_{\mathrm{m}}{ }^{a}$ & $\lambda_{\mathrm{a}, \mathrm{max}, \mathrm{meta}}$ & $\lambda \mathrm{M}$, meta & $\sigma_{\mathrm{p}}{ }^{a}$ & $\lambda_{\mathrm{a}, \mathrm{max}, \text { para }}$ & $\lambda_{\mathrm{M}, \text { meta }}$ \\
\hline$-\mathrm{NH}_{2}$ & -0.16 & 394 & 310 & -0.66 & 373 & 373 \\
\hline$-\mathrm{N}\left(\mathrm{CH}_{3}\right)_{2}$ & -0.16 & 429 & 315 & -0.83 & 382 & 382 \\
\hline$-\mathrm{CH}_{3}$ & -0.07 & 312 & 312 & -0.17 & 333 & 312 \\
\hline$-\mathrm{H}$ & 0 & 316 & 316 & 0 & 341 & 309 \\
\hline$-\mathrm{OH}$ & 0.12 & 341 & 308 & -0.37 & 333 & 333 \\
\hline$-\mathrm{OCH}_{3}$ & 0.12 & 309 & 309 & -0.27 & 342 & 342 \\
\hline$-\mathrm{COOH}$ & 0.37 & 342 & 307 & 0.45 & 350 & 304 \\
\hline$-\mathrm{COCH}_{3}$ & 0.38 & 339 & 307 & 0.5 & 351 & 311 \\
\hline$-\mathrm{CN}$ & 0.56 & 346 & 304 & 0.66 & 362 & 303 \\
\hline$-\mathrm{NO}_{2}$ & 0.71 & 344 & 298 & 0.78 & 416 & 354 \\
\hline
\end{tabular}


Text S1. Derivation of the extent of reaction formula for the estimation of inter molecular charge transfer interactions

The extent of reaction table that corresponds to the equilibrium reaction $\mathrm{A}+\mathrm{D} \rightleftharpoons \mathrm{CT}$ is presented in Table S5:

Table S6. Extent of reaction table
[A]
[D]
[CT]

\begin{tabular}{lccc}
\hline $\mathrm{t}_{0}$ & {$[\mathrm{~A}]_{0}$} & {$[\mathrm{D}]_{0}$} & 0 \\
$\mathrm{t}$ & {$[\mathrm{A}]_{0}-\mathrm{x}$} & {$[\mathrm{D}]_{0}-\mathrm{x}$} & $\mathrm{x}$
\end{tabular}

Where $[\mathrm{A}]_{0}$ and $[\mathrm{D}]_{0}$ are the initial DOM electron acceptor and donor moieties respectively, $[\mathrm{A}]=[\mathrm{A}]_{0}-x,[\mathrm{D}]=[\mathrm{D}]_{0}-x$ and $[\mathrm{CT}]=x$ the concentrations of electron acceptor, donor and inter molecular charge-transfer complexes respectively.

The equilibrium constant $K$ is given by:

$K=\frac{x}{\left([\mathrm{~A}]_{0}-x\right)\left([\mathrm{D}]_{0}-x\right)}$

Equation $\mathrm{S} 1$ can be reorganized to give equation S2:

$x^{2}+x\left(-1 / K-[\mathrm{A}]_{0}-[\mathrm{D}]_{0}\right)+[\mathrm{A}]_{0}[\mathrm{D}]_{0}=0$

Equation $\mathrm{S} 2$ can be solved using the quadratic equation to give:

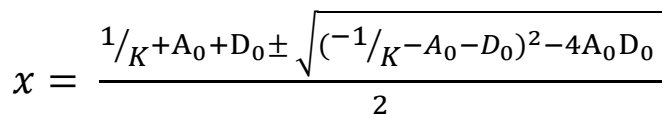

The negative square root solution must be selected to have $\mathrm{x}$ solutions between 0 and 1 . The resulting equation is:

$x=\frac{1 / K+\mathrm{A}_{0}+\mathrm{D}_{0}-\sqrt{\left(-1 / K^{-}-A_{0}-D_{0}\right)^{2}-4 \mathrm{~A}_{0} \mathrm{D}_{0}}}{2}$

Table S7. Evaluation of inter molecular charge transfer interactions

\begin{tabular}{lccccc} 
Group & Ratio $[\mathbf{A}]_{\mathbf{0}} /[\mathbf{D}]_{\mathbf{0}}$ & $\boldsymbol{K}$ & {$[\mathbf{D}]_{\mathbf{0}}$} & $\boldsymbol{x}$ & {$[\mathbf{C T}] /[\mathbf{D}]_{\mathbf{0}}{ }^{\boldsymbol{a}}$} \\
\hline $\mathrm{A}$ & 0.01 & 1 & 0.01 & $9.9 \times 10^{-7}$ & $9.9 \times 10^{-5}$ \\
& 0.01 & 1 & 0.1 & $9.1 \times 10^{-5}$ & $9.1 \times 10^{-4}$ \\
& 0.01 & 1 & 1 & $5.0 \times 10^{-6}$ & $5.0 \times 10^{-3}$ \\
B & 0.01 & 10 & 0.01 & $9.1 \times 10^{-6}$ & $9.1 \times 10^{-4}$ \\
& 0.01 & 10 & 0.1 & $5.0 \times 10^{-4}$ & $5.0 \times 10^{-3}$
\end{tabular}




\begin{tabular}{|c|c|c|c|c|c|}
\hline & 0.01 & 10 & 1 & $9.1 \times 10^{-3}$ & $9.1 \times 10^{-3}$ \\
\hline \multirow[t]{3}{*}{$\mathrm{C}$} & 0.01 & 500 & 0.01 & $8.3 \times 10^{-5}$ & $8.3 \times 10^{-3}$ \\
\hline & 0.01 & 500 & 0.1 & $9.8 \times 10^{-4}$ & $9.8 \times 10^{-3}$ \\
\hline & 0.01 & 500 & 1 & $10.0 \times 10^{-3}$ & $1.0 \times 10^{-2}$ \\
\hline \multirow[t]{3}{*}{ D } & 0.01 & 1000 & 0.01 & $9.1 \times 10^{-5}$ & $9.1 \times 10^{-3}$ \\
\hline & 0.01 & 1000 & 0.1 & $9.9 \times 10^{-4}$ & $9.9 \times 10^{-3}$ \\
\hline & 0.01 & 1000 & 1 & $10.0 \times 10^{-3}$ & $1.0 \times 10^{-2}$ \\
\hline \multirow[t]{3}{*}{$\mathrm{E}$} & 0.1 & 1 & 0.01 & $9.9 \times 10^{-6}$ & $9.9 \times 10^{-4}$ \\
\hline & 0.1 & 1 & 0.1 & $9.0 \times 10^{-4}$ & $9.0 \times 10^{-3}$ \\
\hline & 0.1 & 1 & 1 & $4.9 \times 10^{-2}$ & $4.9 \times 10^{-2}$ \\
\hline \multirow[t]{3}{*}{$\mathrm{F}$} & 0.1 & 10 & 0.01 & $9.0 \times 10^{-5}$ & $9.0 \times 10^{-3}$ \\
\hline & 0.1 & 10 & 0.1 & $4.9 \times 10^{-3}$ & $4.9 \times 10^{-2}$ \\
\hline & 0.1 & 10 & 1 & $9.0 \times 10^{-2}$ & $9.0 \times 10^{-2}$ \\
\hline \multirow[t]{3}{*}{ G } & 0.1 & 500 & 0.01 & $8.2 \times 10^{-4}$ & $8.2 \times 10^{-2}$ \\
\hline & 0.1 & 500 & 0.1 & $9.8 \times 10^{-3}$ & $9.8 \times 10^{-2}$ \\
\hline & 0.1 & 500 & 1 & $1.0 \times 10^{-1}$ & $1.0 \times 10^{-1}$ \\
\hline \multirow[t]{3}{*}{$\mathrm{H}$} & 0.1 & 1000 & 0.01 & $9.0 \times 10^{-4}$ & $9.0 \times 10^{-2}$ \\
\hline & 0.1 & 1000 & 0.1 & $9.9 \times 10^{-3}$ & $9.9 \times 10^{-2}$ \\
\hline & 0.1 & 1000 & 1 & $1.0 \times 10^{-1}$ & $1.0 \times 10^{-1}$ \\
\hline \multirow[t]{3}{*}{ I } & 1 & 1 & 0.01 & $9.8 \times 10^{-5}$ & $9.8 \times 10^{-3}$ \\
\hline & 1 & 1 & 0.1 & $8.4 \times 10^{-3}$ & $8.4 \times 10^{-2}$ \\
\hline & 1 & 1 & 1 & $3.8 \times 10^{-1}$ & $3.8 \times 10^{-1}$ \\
\hline \multirow[t]{3}{*}{$\mathrm{J}$} & 1 & 10 & 0.01 & $8.4 \times 10^{-4}$ & $8.4 \times 10^{-2}$ \\
\hline & 1 & 10 & 0.1 & $3.8 \times 10^{-2}$ & $3.8 \times 10^{-1}$ \\
\hline & 1 & 10 & 1 & $7.3 \times 10^{-1}$ & $7.3 \times 10^{-1}$ \\
\hline \multirow[t]{3}{*}{ K } & 1 & 500 & 0.01 & $6.4 \times 10^{-3}$ & $6.4 \times 10^{-1}$ \\
\hline & 1 & 500 & 0.1 & $8.7 \times 10^{-2}$ & $8.7 \times 10^{-1}$ \\
\hline & 1 & 500 & 1 & $9.6 \times 10^{-1}$ & $9.6 \times 10^{-1}$ \\
\hline \multirow[t]{3}{*}{$\mathrm{L}$} & 1 & 1000 & 0.01 & $7.3 \times 10^{-3}$ & $7.3 \times 10^{-1}$ \\
\hline & 1 & 1000 & 0.1 & $9.0 \times 10^{-2}$ & $9.0 \times 10^{-1}$ \\
\hline & 1 & 1000 & 1 & $9.7 \times 10^{-1}$ & $9.7 \times 10^{-1}$ \\
\hline \multirow[t]{3}{*}{ M } & 1 & 5000 & 0.01 & $8.7 \times 10^{-3}$ & $8.7 \times 10^{-1}$ \\
\hline & 1 & 5000 & 0.1 & $9.6 \times 10^{-2}$ & $9.6 \times 10^{-1}$ \\
\hline & 1 & 5000 & 1 & $9.9 \times 10^{-1}$ & $9.9 \times 10^{-1}$ \\
\hline \multirow[t]{3}{*}{$\mathrm{N}$} & 1 & 10000 & 0.01 & $9.0 \times 10^{-3}$ & $9.0 \times 10^{-1}$ \\
\hline & 1 & 10000 & 0.1 & $9.7 \times 10^{-2}$ & $9.7 \times 10^{-1}$ \\
\hline & 1 & 10000 & 1 & $9.9 \times 10^{-1}$ & $9.9 \times 10^{-1}$ \\
\hline
\end{tabular}

Note: ${ }^{a}$ For CT to be $[\mathrm{DOM}]$ independent, this ratio has to be constant for a group ([DOM] being proportional to $\left.[\mathrm{D}]_{0}\right)$. 


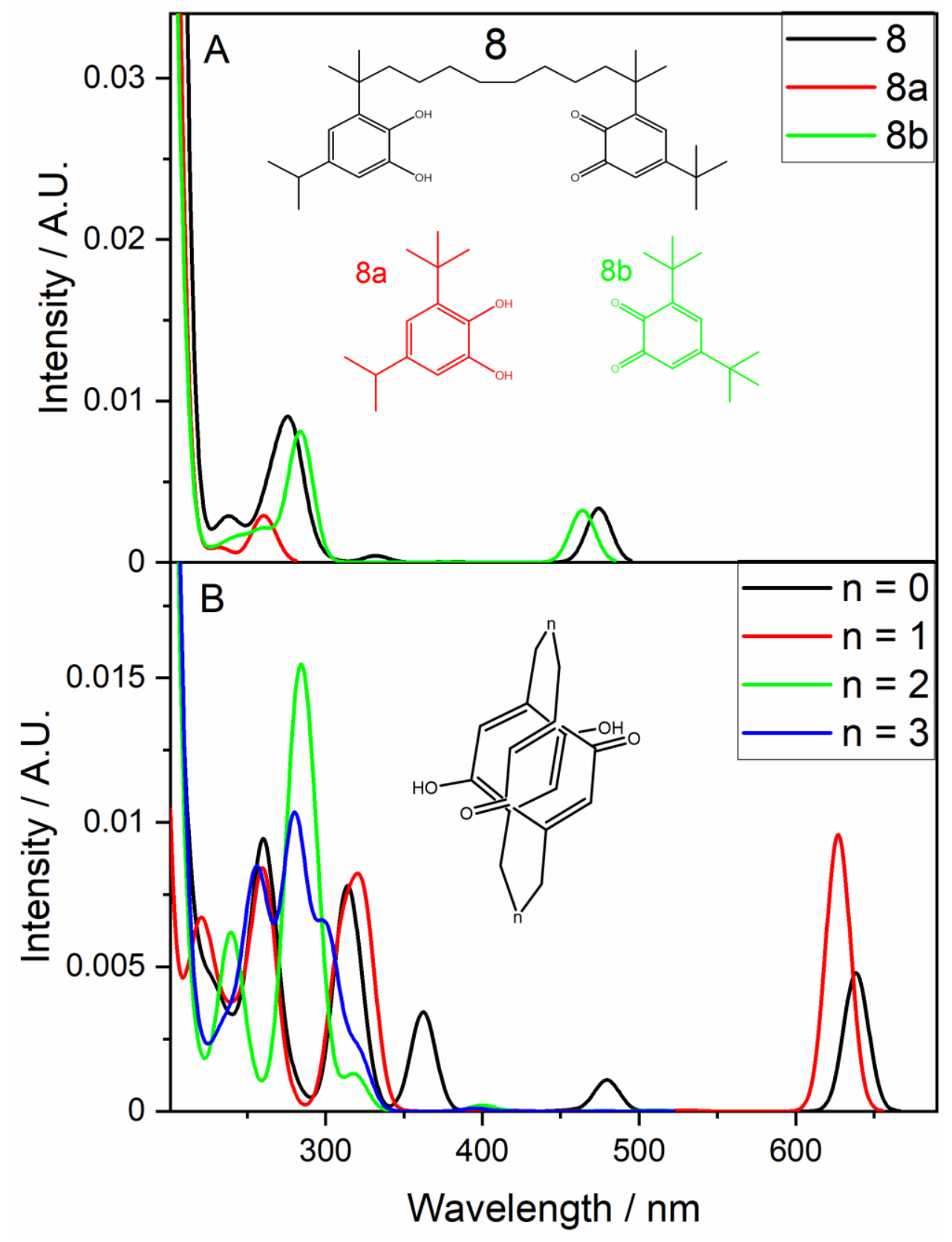

Figure S3. Additional compounds investigated for intra-molecular charge transfer interactions. Note, the original molecule presented in the main text have $n=1$. 


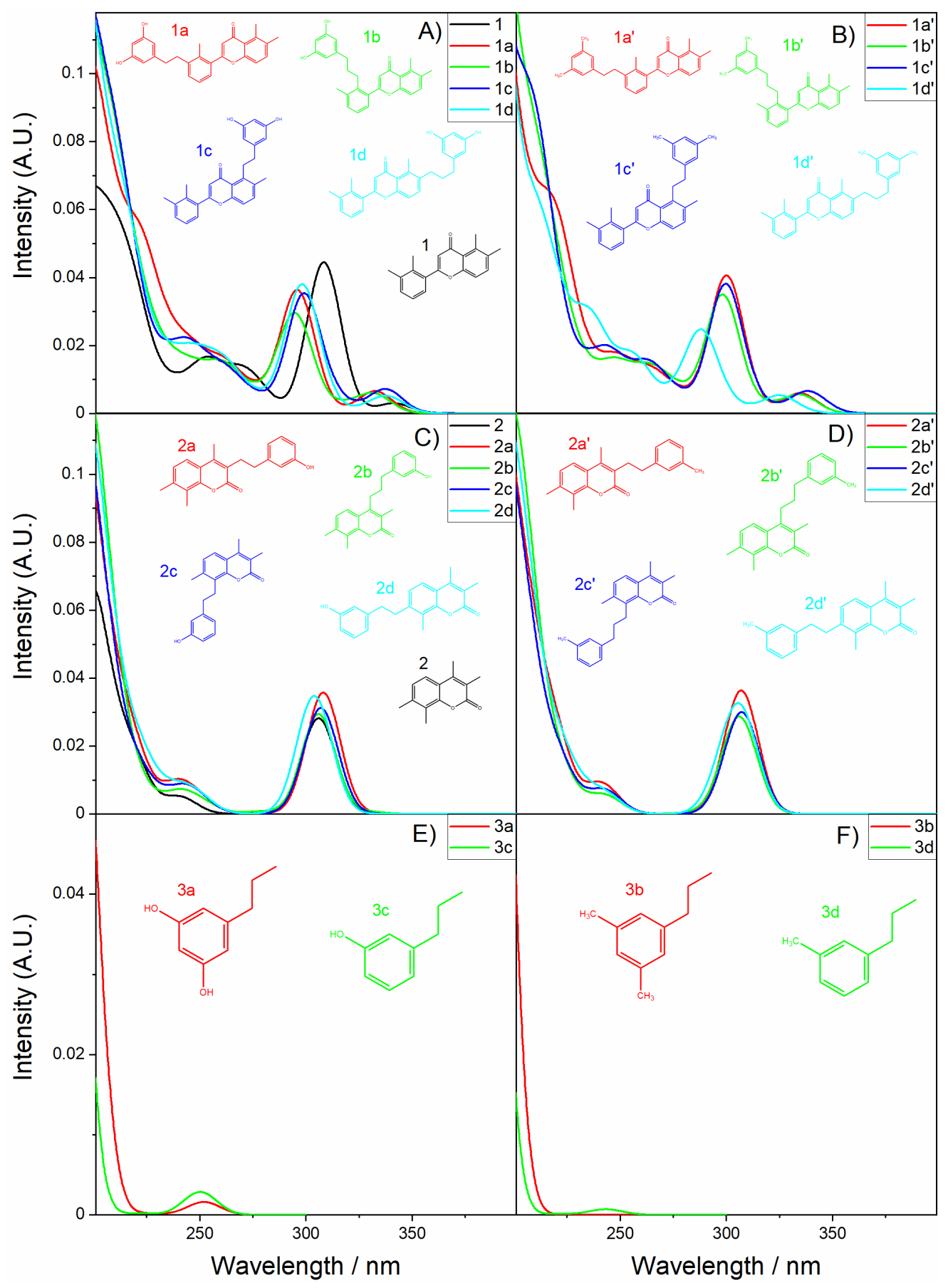

Figure S4. Additional flavone and coumarin backbone molecules grafted with hydroxy or dihydroxy-benzene groups. Note, the molecules numbers correspond to Table S2 numbering and differs from Figure 2, main text. 


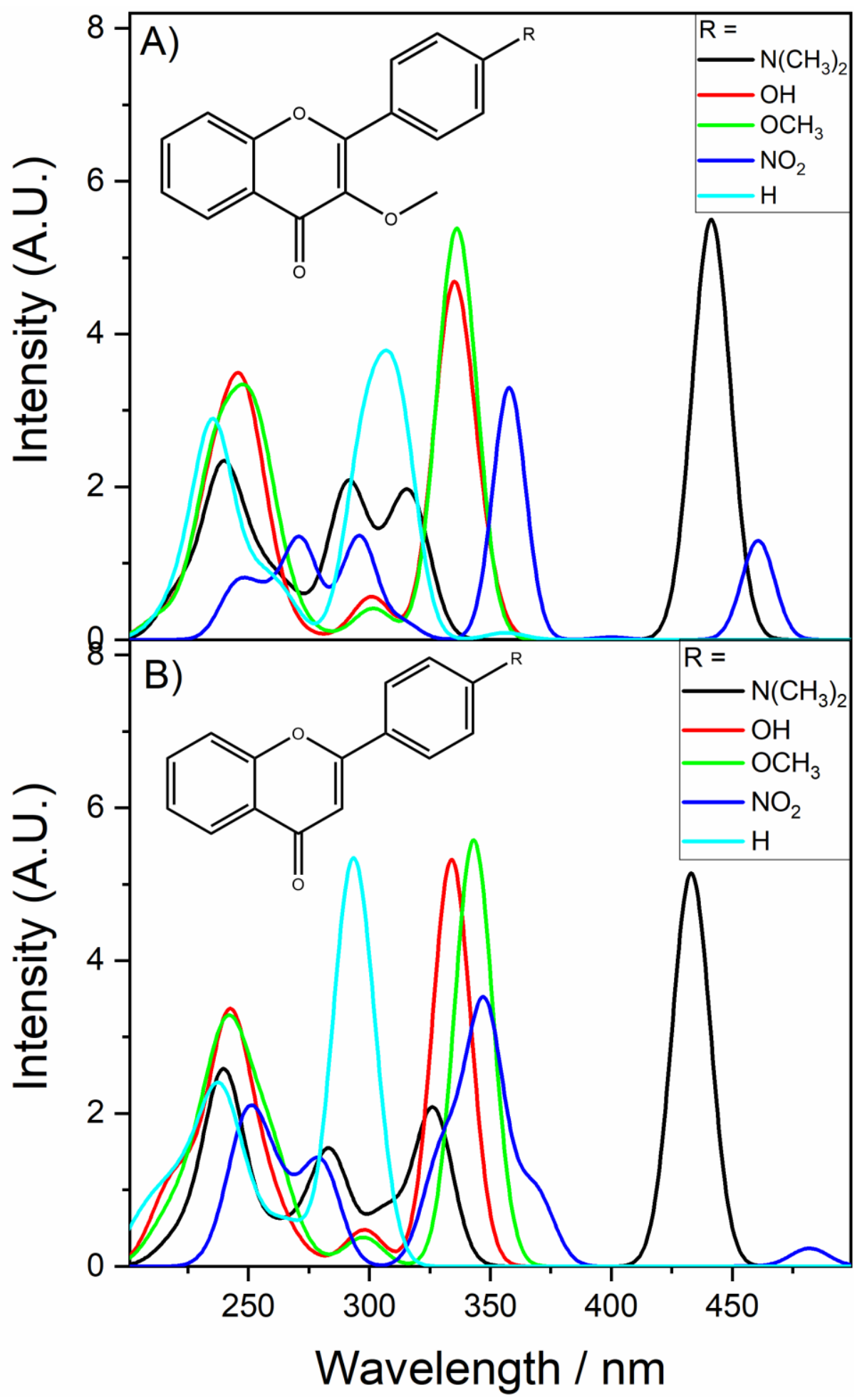

Figure S5. Additional molecules investigated for conjugated intra molecular charge-transfer (CT) interactions. 
Table S8. Calculated charge transfer (CT) contribution to the lowest singlet excited state of flavone derivatives

\begin{tabular}{|c|c|c|c|c|}
\hline Molecule & $-\mathrm{R}$ & $\mathrm{dE}(\mathrm{eV})$ & $\begin{array}{c}\text { Oscillator } \\
\text { strength }\end{array}$ & CT $(\%)$ \\
\hline & $-\mathrm{N}\left(\mathrm{CH}_{3}\right)_{2}$ & 2.86 & 0.16 & 71 \\
\hline & $-\mathrm{OH}$ & 3.71 & 0.13 & 60 \\
\hline & $-\mathrm{OCH}_{3}$ & 3.61 & 0.17 & 62 \\
\hline & $-\mathrm{NO}_{2}$ & 2.57 & 0.02 & 81 \\
\hline & $-\mathrm{H}$ & 4.14 & 0.03 & 37 \\
\hline & $-\mathrm{N}\left(\mathrm{CH}_{3}\right)_{2}$ & 2.72 & 0.10 & 67 \\
\hline & $-\mathrm{OH}$ & 3.44 & 0.06 & 48 \\
\hline & $-\mathrm{OCH}_{3}$ & 3.37 & 0.08 & 51 \\
\hline & $-\mathrm{NO}_{2}$ & 2.43 & 0.06 & 76 \\
\hline & $-\mathrm{H}$ & 3.71 & 0.05 & 35 \\
\hline & $-\mathrm{N}\left(\mathrm{CH}_{3}\right)_{2}$ & 2.81 & 0.10 & 69 \\
\hline & $-\mathrm{OH}$ & 3.60 & 0.01 & 23 \\
\hline & $-\mathrm{OCH}_{3}$ & 3.66 & 0.04 & 37 \\
\hline & $-\mathrm{NO}_{2}$ & 2.46 & 0.03 & 75 \\
\hline$\prod_{0}$ & $-\mathrm{H}$ & 3.48 & 0.001 & 19 \\
\hline
\end{tabular}


Table S9. Effects of sodium borohydride reduction (BHR) on the absorption maximum ( $\left.\lambda_{\mathrm{a}, \max }\right)$ of the models

\begin{tabular}{|c|c|c|c|c|c|c|}
\hline \multirow[b]{2}{*}{ Model \# } & \multicolumn{3}{|c|}{$p H 1$} & \multicolumn{3}{|c|}{ pH 5} \\
\hline & $\begin{array}{c}\lambda_{\mathrm{a}, \max } \text { before } \\
\mathrm{BHR}^{a}\end{array}$ & $\begin{array}{c}\lambda_{\mathrm{a}, \max } \text { after } \\
\mathrm{BHR}^{a}\end{array}$ & $\begin{array}{c}\text { Average } \lambda_{\text {a,max }} \\
\text { shift } b /(\boldsymbol{c})\end{array}$ & $\begin{array}{c}\lambda \mathrm{a} \text {,max before } \\
\text { BHR }^{a}\end{array}$ & $\begin{array}{l}\lambda \mathrm{a} \text {,max after } \\
\mathrm{BHR}^{a}\end{array}$ & 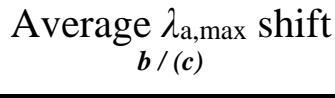 \\
\hline 1 & 471 & 483 & $15 \pm 7(28 \pm 12)$ & 557 & 460 & $56 \pm 16(91 \pm 15)$ \\
\hline 2 & 427 & 337 & $29 \pm 12(60 \pm 20)$ & 611 & 374 & $39 \pm 18(92 \pm 36)$ \\
\hline 3 & 411 & 460 & $25 \pm 8(33 \pm 9)$ & 457 & 369 & $44 \pm 9(53 \pm 8)$ \\
\hline 4 & 527 & 503 & $20 \pm 11(22 \pm 13)$ & 557 & 488 & $65 \pm 20(87 \pm 18)$ \\
\hline 5 & 495 & 483 & $34 \pm 17(47 \pm 23)$ & 495 & 435 & $67 \pm 18(73 \pm 18)$ \\
\hline Global model & 527 & 503 & $25 \pm 5(40 \pm 7)$ & 611 & 488 & $51 \pm 8(78 \pm 9)$ \\
\hline
\end{tabular}

Note: ${ }^{a}$ calculated on the model UV-Vis absorbance spectra ${ }^{b}$ calculated as a mean of the $\lambda_{\mathrm{a}, \max }$ shifts of individual molecules in the model, mean \pm standard error of the mean, ${ }^{c}$ value calculated as mean of $\lambda_{\mathrm{a} \text {,max }}$ shifts of individual molecules present in the model excluding molecules, that had $\lambda_{\mathrm{a} \text {,max }}$ shift $=0$. 


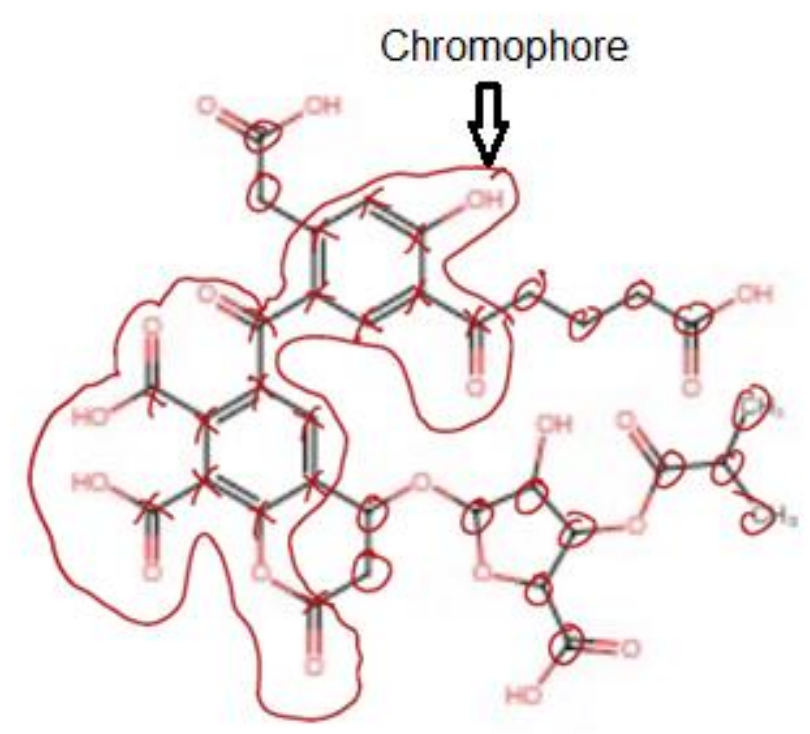

- Carbon atoms that involved in
chromophore unit (functional group)

- Carbon atoms that do not absorbe light above $250 \mathrm{~nm}$

Figure S6. Example of calculation of the chromophoric part of a molecule. Number of carbon atoms in chromophore is 17 . Overall number of atoms is 34 . Percentage of $\mathrm{cDOM}=17 / 34 * 100 \%=50 \%$

Table S10. Evaluation of DOM chromophoric carbon fraction

\begin{tabular}{lccc} 
model & $\begin{array}{c}\text { chromophore } \\
\text { carbon }\end{array}$ & $\begin{array}{c}\text { total } \\
\text { carbon }\end{array}$ & ratio \\
\hline 1 & 102 & 187 & 0.55 \\
2 & 93 & 174 & 0.53 \\
3 & 98 & 190 & 0.52 \\
4 & 94 & 183 & 0.51 \\
5 & 108 & 196 & 0.55 \\
global & 495 & 930 & 0.53
\end{tabular}




\section{References for the Supplementary Information}

(1) Vialykh, E. A.; McKay, G.; Rosario-Ortiz, F. L. Computational Assessment of the Three-Dimensional Configuration of Dissolved Organic Matter Chromophores and Influence on Absorption Spectra. Environmental Science \& Technology 2020, 54 (24), 15904-15913. DOI: 10.1021/acs.est.0c05860.

(2) Hansch, C.; Leo, A.; Taft, R. W. A Survey of Hammett Substituent Constants and Resonance and Field Parameters. Chemical Reviews 1991, 91 (2), 165-195. DOI: DOI 10.1021/cr00002a004. 